\title{
THE COSMOLOGICAL IMPACT OF LUMINOUS TEV BLAZARS I: IMPLICATIONS OF PLASMA INSTABILITIES FOR THE INTERGALACTIC MAGNETIC FIELD AND EXTRAGALACTIC GAMMA-RAY BACKGROUND
}

\author{
Avery E. Broderick ${ }^{1,2,3}$, Philip ChAnG ${ }^{1,4}$, AND Christoph PFrommer ${ }^{5,1}$ \\ ${ }^{1}$ Canadian Institute for Theoretical Astrophysics, 60 St. George Street, Toronto, ON M5S 3H8, Canada; aeb@ @ita.utoronto.ca, pchang@cita.utoronto.ca \\ ${ }^{2}$ Perimeter Institute for Theoretical Physics, 31 Caroline Street North, Waterloo, ON, N2L 2Y5, Canada \\ ${ }^{3}$ Department of Physics and Astronomy, University of Waterloo, 200 University Avenue West, Waterloo, ON, N2L 3G1, Canada \\ ${ }^{4}$ Department of Physics, University of Wisconsin-Milwaukee, 1900 E. Kenwood Boulevard, Milwaukee, WI 53211, USA \\ ${ }^{5}$ Heidelberg Institute for Theoretical Studies, Schloss-Wolfsbrunnenweg 35, D-69118 Heidelberg, Germany; christoph.pfrommer@h-its.org \\ Draft version October 31, 2018
}

\begin{abstract}
Inverse-Compton cascades initiated by energetic gamma rays $(E \gtrsim 100 \mathrm{GeV})$ enhance the $\mathrm{GeV}$ emission from bright, extragalactic $\mathrm{TeV}$ sources. The absence of this emission from bright $\mathrm{TeV}$ blazars has been used to constrain the intergalactic magnetic field (IGMF), and the stringent limits placed upon the unresolved extragalactic gammaray background (EGRB) by Fermi has been used to argue against a large number of such objects at high redshifts. However, these are predicated upon the assumption that inverse-Compton scattering is the primary energy-loss mechanism for the ultra-relativistic pairs produced by the annihilation of the energetic gamma rays on extragalactic background light photons. Here we show that for sufficiently bright $\mathrm{TeV}$ sources (isotropic-equivalent luminosities $\gtrsim 10^{42} \mathrm{erg} \mathrm{s}^{-1}$ ) plasma beam instabilities, specifically the "oblique" instability, present a plausible mechanism by which the energy of these pairs can be dissipated locally, heating the intergalactic medium. Since these instabilities typically grow on timescales short in comparison to the inverse-Compton cooling rate, they necessarily suppress the inverse-Compton cascades. As a consequence, this places a severe constraint upon efforts to limit the IGMF from the lack of a discernible GeV bump in TeV sources. Similarly, it considerably weakens the Fermi limits upon the evolution of blazar populations. Specifically, we construct a TeV-blazar luminosity function from those objects presently observed and find that it is very well described by the quasar luminosity function at $z \sim 0.1$, shifted to lower luminosities and number densities, suggesting that both classes of sources are regulated by similar processes. Extending this relationship to higher redshifts, we show that the magnitude and shape of the EGRB above $\sim 10 \mathrm{GeV}$ is naturally reproduced with this particular example of a rapidly evolving TeV-blazar luminosity function.

Subject headings: BL Lacertae objects: general - gamma rays: general - instabilities - magnetic fields - plasmas - radiative mechanisms: non-thermal
\end{abstract}

\section{INTRODUCTION}

Imaging atmospheric Cerenkov telescopes, such as H.E.S.S., VERITAS, and MAGIC, ${ }^{1}$ have opened the very-high energy gamma-ray (VHEGR, $E \geq 100 \mathrm{GeV}$ ) sky, finding a Universe populated by a variety of energetic, VHEGR sources. While the majority of observed VHEGR sources are Galactic in origin (e.g., supernova remnants, etc.), the extragalactic contribution is dominated by a subset of blazars. There are presently 46 extragalactic TeV sources known ${ }^{2}$, of which 28 have well defined spectral energy distributions (SEDs), and are collected in Table 1. Of these 28 well-studied objects, 24 are blazars, implying that blazars make up an overwhelming majority of the bright VHEGR sources.

All of the extragalactic VHEGR emitters are relatively nearby, with $z \lesssim 0.5$ generally, and $z \sim 0.1$ typical. This is a result of the large opacity of the Universe to $\mathrm{TeV}$ photons, which annihilate upon soft photons in the extragalactic background light (EBL), producing pairs (see, e.g., Gould \& Schréder 1967; Salamon \& Stecker 1998; Neronov \& Semikoz 2009). Typical mean free paths of VHEGRs range from $30 \mathrm{Mpc}$ to $1 \mathrm{Gpc}$ depending upon gamma-ray energy and source redshift,

\footnotetext{
${ }^{1}$ High Energy Stereoscopic System, Major Atmospheric Gamma Imaging Cerenkov Telescope, Very Energetic Radiation Imaging Telescope Array Sys-
tem.

${ }^{2}$ See http://www.mppmu.mpg.de/ rwagner/sources/ for an up-to-date list.
}

and thus the absence of high-redshift VHEGR sources is not unexpected.

The pairs produced by VHEGR annihilation are necessarily ultrarelativistic, with typical Lorentz factors of $10^{5}-10^{7}$. The standard assumption is that these pairs lose energy almost exclusively through inverse-Compton scattering the cosmic microwave background (CMB) and EBL. When the up-scattered gamma-ray is itself a VHEGR the process repeats, creating a second generation of pairs and up-scattering additional photons. The result is an inverse-Compton cascade (ICC) depositing the energy of the original VHEGR in gamma-rays with energies $\lesssim 100 \mathrm{GeV}$. This places the ICC gamma rays in the LAT bands of Fermi, and thus Fermi has played a central role in constraining the VHEGR emission of high-redshift blazars.

Based upon Fermi observations of $z \simeq 0.1 \mathrm{TeV}$ sources, a number of authors have now published estimated lower bounds upon the intergalactic magnetic field (IGMF; see, e.g., Neronov \& Vovk 2010; Tavecchio et al. 2010, 2011; Dermer et al. 2011; Taylor et al. 2011; Takahashi et al. 2012; Dolag et al. 2011). Typical numbers range from $10^{-19} \mathrm{G}$ to $10^{-15} \mathrm{G}$, with the latter values being of astrophysical interest in the context of the formation of galactic fields ${ }^{3}$. These limits on the IGMF arise from the lack of the GeV bump associated with the ICC of the blazar

\footnotetext{
${ }^{3}$ After contraction and a handful of windings, $\mathrm{nG}$ field strengths can be produced from an IGMF of $\sim 10^{-15} \mathrm{G}$.
} 
Table 1

List of TeV Sources with Measured Spectral Properties in Decreasing $100 \mathrm{GeV}-10 \mathrm{TeV}$ Flux Order

\begin{tabular}{|c|c|c|c|c|c|c|c|c|c|c|c|}
\hline Name & $z$ & $D_{C}{ }^{\mathrm{a}}$ & $f_{0} \mathrm{~b}$ & $E_{0}{ }^{\mathrm{c}}$ & $\alpha^{\mathrm{d}}$ & $F^{\mathrm{e}}$ & $\log _{10} L^{\mathrm{f}}$ & $\hat{\alpha}^{\mathrm{g}}$ & $\Delta t^{\mathrm{h}}$ & Class $^{i}$ & Reference \\
\hline Mkn 421 & 0.030 & 129 & 68 & 1 & 3.32 & $1.7 \times 10^{3}$ & 45.6 & 3.15 & $2.8 \times 10^{2}$ & $\mathrm{H}$ & Chandra et al. (2010) \\
\hline 1ES $1959+650$ & 0.047 & 201 & 78 & 1 & 3.18 & $1.6 \times 10^{3}$ & 45.9 & 2.90 & 85 & $\mathrm{H}$ & Aharonian et al. (2003) \\
\hline 1ES $2344+514$ & 0.044 & 190 & 120 & 0.5 & 2.95 & $2.3 \times 10^{2}$ & 45.0 & 2.82 & $5.0 \times 10^{2}$ & $\mathrm{H}$ & Albert et al. (2007c) \\
\hline Mkn $501^{\mathrm{j}}$ & 0.034 & 150 & 8.7 & 1 & 2.58 & 85 & 44.4 & 2.39 & $1.6 \times 10^{3}$ & $\mathrm{H}$ & Huang et al. (2009) \\
\hline 3C 279 & 0.536 & 2000 & 520 & 0.2 & 4.11 & 68 & 46.9 & 2.53 & 2.0 & $\mathrm{Q}$ & MAGIC Collaboration et al. (2008a) \\
\hline PKS 2155-304 & 0.116 & 490 & 1.81 & 1 & 3.53 & 64 & 45.4 & 2.75 & $3.3 \times 10^{2}$ & $\mathrm{H}$ & HESS Collaboration et al. (2010) \\
\hline PG $1553+113$ & $>0.09$ & $>380$ & 46.8 & 0.3 & 4.46 & 41 & $>44.9$ & $<4.29$ & $<5.7 \times 10^{3}$ & $\mathrm{H}$ & Aharonian et al. (2008b) \\
\hline W Comae & 0.102 & 430 & 20 & 0.4 & 3.68 & 31 & 44.9 & 3.41 & $1.3 \times 10^{3}$ & I & Acciari et al. (2009b) \\
\hline $3 \mathrm{C} 66 \mathrm{~A}$ & 0.444 & 1700 & 40 & 0.3 & 4.1 & 28 & 46.3 & 2.43 & 13 & $\mathrm{I}$ & Acciari et al. (2009c) \\
\hline 1ES $1011+496$ & 0.212 & 870 & 200 & 0.2 & 4 & 26 & 45.5 & 3.66 & $2.5 \times 10^{2}$ & $\mathrm{H}$ & Albert et al. (2007b) \\
\hline 1ES $1218+304 \mathrm{j}$ & 0.182 & 750 & 11.5 & 0.5 & 3.07 & 24 & 45.4 & 2.37 & $1.0 \times 10^{2}$ & $\mathrm{H}$ & Acciari et al. (2010a) \\
\hline Mkn 180 & 0.045 & 190 & 45 & 0.3 & 3.25 & 20 & 44.0 & 3.17 & $8.2 \times 10^{3}$ & $\mathrm{H}$ & Albert et al. (2006) \\
\hline $1 \mathrm{H} 1426+428$ & 0.129 & 540 & 2 & 1 & 2.6 & 20 & 45.0 & 1.71 & $2.1 \times 10^{2}$ & $\mathrm{H}$ & Aharonian et al. (2002) \\
\hline RGB J0710+591 j & 0.125 & 520 & 1.36 & 1 & 2.69 & 15 & 44.8 & 1.83 & $3.5 \times 10^{2}$ & $\mathrm{H}$ & Acciari et al. (2010c) \\
\hline 1ES $0806+524$ & 0.138 & 580 & 6.8 & 0.4 & 3.6 & 10 & 44.7 & 3.21 & $1.4 \times 10^{3}$ & $\mathrm{H}$ & Acciari et al. (2009a) \\
\hline RGB J0152+017 j & 0.080 & 340 & 0.57 & 1 & 2.95 & 8.5 & 44.1 & 2.45 & $3.0 \times 10^{3}$ & $\mathrm{H}$ & Aharonian et al. (2008a) \\
\hline $1 E S 1101-232^{j}$ & 0.186 & 770 & 0.56 & 1 & 2.94 & 8.2 & 44.9 & 1.50 & $2.3 \times 10^{2}$ & $\mathrm{H}$ & Aharonian et al. (2007a) \\
\hline 1ES $0347-121 \mathrm{j}$ & 0.185 & 770 & 0.45 & 1 & 3.1 & 8.2 & 44.9 & 1.67 & $2.9 \times 10^{2}$ & $\mathrm{H}$ & Aharonian et al. (2007b) \\
\hline IC 310 & 0.019 & 83 & 1.1 & 1 & 2.0 & 8.1 & 42.8 & 1.90 & $4.8 \times 10^{4}$ & $\mathrm{H}$ & Aleksić et al. (2010) \\
\hline PKS 2005-489 & 0.071 & 300 & 0.1 & 1 & 4.0 & 8.0 & 44.0 & 3.56 & $2.3 \times 10^{4}$ & $\mathrm{H}$ & Aharonian et al. (2005) \\
\hline MAGIC J0223+430 & - & - & 17.4 & 0.3 & 3.1 & 7.6 & - & $<3.1$ & - & $\mathrm{R}$ & Aliu et al. (2009) \\
\hline $1 E S 0229+200^{j}$ & 0.140 & 590 & 0.7 & 1 & 2.5 & 6.4 & 44.5 & 1.51 & $4.7 \times 10^{2}$ & $\mathrm{H}$ & Aharonian et al. (2007c) \\
\hline PKS $1424+240$ & $<0.66$ & $<2400$ & 51 & 0.2 & 3.8 & 6.3 & $<46.1$ & $>1.42$ & 4.0 & $\mathrm{I}$ & Acciari et al. (2010b) \\
\hline M87 & 0.0044 & 19 & 0.74 & 1 & 2.31 & 5.9 & 41.4 & 2.29 & $1.5 \times 10^{6}$ & $\mathrm{R}$ & Acciari et al. (2008) \\
\hline BL Lacertae & 0.069 & 290 & 0.3 & 1 & 3.09 & 5.4 & 43.8 & 2.67 & $8.4 \times 10^{3}$ & $\mathrm{~L}$ & Albert et al. (2007a) \\
\hline Н 2356-309 & 0.165 & 690 & 0.3 & 1 & 3.09 & 5.4 & 44.6 & 1.86 & $6.5 \times 10^{2}$ & $\mathrm{H}$ & Aharonian et al. (2006) \\
\hline PKS 0548-322 j & 0.069 & 290 & 0.3 & 1 & 2.86 & 4.0 & 43.7 & 2.44 & $8.4 \times 10^{3}$ & $\mathrm{H}$ & Aharonian et al. (2010) \\
\hline Centaurus A & 0.0028 & 12 & 0.245 & 1 & 2.73 & 2.8 & 40.7 & 2.72 & $1.2 \times 10^{7}$ & $\mathrm{R}$ & Raue et al. (2010) \\
\hline
\end{tabular}

a Comoving distance in units of $\mathrm{Mpc}$

${ }^{\mathrm{b}}$ Normalization of the observed photon spectrum that we assume to be of the form $d N / d E=f_{0}\left(E / E_{0}\right)^{-\alpha}$, in units of $10^{-12} \mathrm{~cm}^{-2} \mathrm{~s}^{-1} \mathrm{TeV}^{-1}$

c Energy at which we normalize the spectrum, in units of TeV

d Observed spectral index at $E_{0}$

e Integrated energy flux between $100 \mathrm{GeV}$ and $10 \mathrm{TeV}$, in units of $10^{-12} \mathrm{erg} \mathrm{cm}^{-2} \mathrm{~s}^{-1}$

f Inferred isotropic integrated luminosity between $100 \mathrm{GeV}$ and $10 \mathrm{TeV}$, in units of $\mathrm{erg} \mathrm{s}^{-1}$

$\mathrm{g}$ Inferred intrinsic spectral index at $E_{0}$

h Time delay after which plasma beam instabilities dominate inverse-Compton cooling, in units of yr

${ }^{i}$ H, I, L, Q, and R correspond to high-energy, intermediate-energy, low-energy peaked BL Lacs, flat spectrum radio quasars, and radio galaxies of Faranoff-Riley Type I (FR I), respectively.

$\mathrm{j}$ Used to place limits upon the IGMF

$\mathrm{TeV}$ emission, presumably due to the resulting pairs being deflected significantly under the action of the IGMF itself. The wide range in the estimates upon the minimum IGMF is due primarily to different assumptions about the $\mathrm{TeV}$ blazar duty cycle.

Fermi has also provided the most precise estimate of the unresolved extragalactic gamma-ray background (EGRB) for energies between $200 \mathrm{MeV}$ and $100 \mathrm{GeV}$. Since ICCs reprocess the VHEGR emission of distant sources into this band, this has been used to constrain the evolution of the luminosity density of VHEGR sources (see, e.g., Narumoto \& Totani 2006; Kneiske \& Mannheim 2008; Inoue \& Totani 2009; Venters 2010). Generally, it has been found that these cannot have exhibited the dramatic rise in numbers by $z \sim 1-2$ seen in the quasar distribution. That is, the comoving number of blazars must have remained essentially fixed, at odds with both the physical picture underlying these systems and with the observed evolution of similarly accreting systems, i.e., quasars.

Both of these conclusions depend critically upon ICCs dominating the evolution of the ultra-relativistic pairs. However, as we will show, the pairs constitute a cold, highly collimated plasma beam moving through a dense, stationary background, both of which are susceptible to collective plasma phenomena. Such beams are notoriously unstable; for instance, equal den- sity beams typically lose a significant fraction of their energy after propagating distances measured in plasma skin depths of the background plasma. If the VHEGR-generated pairs suffer a similar fate while propagating through the intergalactic medium (IGM), the cooling of the pairs would be dominated by plasma instabilities, thereby quenching the ICCs.

Here we present a plausible alternative mechanism by which the energy in the ultra-relativistic pairs can be extracted. While a variety of potential plasma beam instabilities exist, we find that the most relevant for the VHEGR-produced pair beams is the "oblique" instability (Bret et al. 2004, 2005a; Bret 2009; Bret et al. 2010b; Lemoine \& Pelletier 2010). This is a more virulent cousin of the commonly discussed Weibel and two stream instabilities.

In Section 2 we discuss the formation and properties of the ultra-relativistic pair beam, including limits upon its temperature and density. Section 3 presents growth rates for a variety of plasma instabilities, including the oblique instability. Particular attention is paid to if and when plasma instabilities dominate inverse Compton as a means to dissipate the kinetic energy of the pairs. The resulting implications for studies of the IGMF are described in Section 4, including how such efforts might mitigate the systematic uncertainties arising from plasma cooling. The evolution of the luminosity function of 
VHEGR-emitting blazars is discussed in Section 5, in which we construct a blazar luminosity function based upon that of quasars which is consistent with current $\mathrm{TeV}$ source populations and the Fermi estimates of the EGRB. Finally, conclusions are contained in Section 6.

This is the first in a series of three papers that discuss the potential cosmological impact of the $\mathrm{TeV}$ emission from blazars. Here we provide a plausible mechanism for the local dissipation of the VHEGR luminosity of bright gamma-ray sources. In addition to the particular consequences this has for studies of high-energy gamma-ray phenomenology, it also provides an novel heating process within the IGM. Chang et al. (2012, hereafter Paper II) estimates the magnitude of the new heating term, describes the associated modifications to the thermal history of the IGM, and shows how this can explain some recent observations of the Ly $\alpha$ forest. Pfrommer et al. (2012, hereafter Paper III) considers the impact the new heating term has upon the structure and statistics of galaxy clusters and groups, and upon the ages and properties of dwarf galaxies throughout the Universe, generally finding that blazar heating can help explain outstanding questions in both cases. An additional follow-up paper by Puchwein et al. (2011) shows that when combined with most recent estimates of the evolving photoionizing background and hydrodynamic simulations of cosmological structure formation, the heating by blazars results in excellent quantitative agreement with observations of the mean transmission, one- and two-point statistics, and line width distribution of high-redshift Ly $\alpha$ forest spectra. In particular, these successes depends upon the peculiar properties of the blazar heating via the dissipation of plasma instabilities.

For all of the calculations presented below we assume the WMAP7 cosmology with $h_{0}=0.704, \Omega_{D M}=0.227, \Omega_{B}=$ 0.0456 , and $\Omega_{\Lambda}=0.728$ (Komatsu et al. 2011).

\section{THE FATE OF VERY HIGH ENERGY GAMMA RAYS AND THE} PROPERTIES OF THE RESULTING PAIR BEAM

\subsection{Propagation and Absorption of Very High-Energy Gamma rays}

Sources of VHEGRs are necessarily attenuated by the production of pairs upon interacting with the EBL. Namely, when the energies of the gamma ray $(E)$ and the EBL photon $\left(E_{\mathrm{EBL}}\right)$ exceed the rest mass energy of the $e^{ \pm}$pair in the center of mass frame, i.e., $2 E E_{\mathrm{EBL}}(1-\cos \theta)>4 m_{e}^{2} c^{4}$, where $\theta$ is the relative angle of propagation in the lab frame, an $e^{ \pm}$pair can be produced with Lorentz factor $\gamma \simeq E / 2 m_{e} c^{2}$ (Gould \& Schréder 1967). The optical depth the Universe presents to high-energy gamma rays depends solely upon the energy of the gamma ray and the evolving spectrum of the $\mathrm{EBL}^{4}$. Detailed estimates for the EBL spectrum and its evolution have produced an estimated mean free path of $\mathrm{TeV}$ photons of

$$
D_{\mathrm{pp}}(E, z)=35\left(\frac{E}{1 \mathrm{TeV}}\right)^{-1}\left(\frac{1+z}{2}\right)^{-\zeta} \mathrm{Mpc},
$$

where the redshift evolution is due to that of the EBL alone, is dependent predominately upon the star formation history, and $\zeta=4.5$ for $z<1$ and $\zeta=0$ for $z \geq 1$ (Kneiske et al. 2004; Neronov \& Semikoz 2009) $)^{5}$. The resulting optical depth to

\footnotetext{
${ }^{4}$ As a consequence, careful studies of the absorption of high-energy gamma rays from extragalactic sources has been suggested as a way to probe the EBL (see, e.g., Gould \& Schréder 1967; Stecker et al. 1992; Oh 2001).

${ }^{5}$ Despite the fact that the EBL contribution from starbursts peaks at $z=3$ and declines rapidly afterward, galaxies and Type 1 active galactic nuclei compen-
}

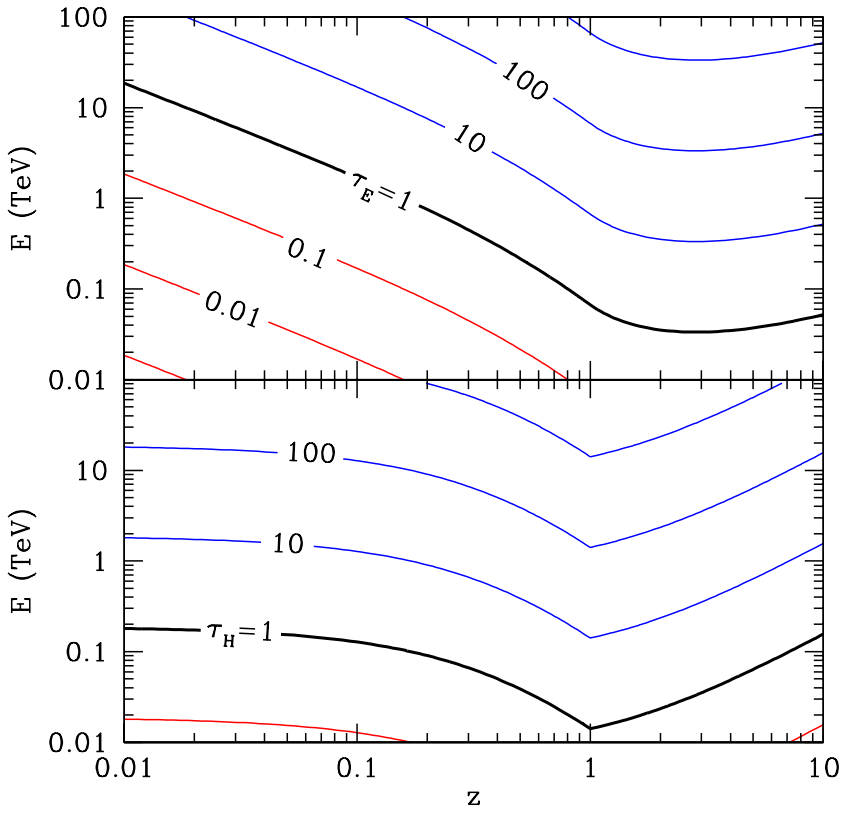

Figure 1. Top: Pair-production optical depth of a gamma-ray photon emitted with energy $E$ originating at redshift $z$ as it propagates to the Earth, $\tau_{E}$. Bottom: Pair-production optical depth of a gamma ray of energy $E$ propagating across a Hubble distance at redshift $z, \tau_{H}$. In both cases the thick black line shows $\tau$ of unity, blue and red lines show optically thick and thin regions of the parameter space, respectively. The sudden break in the contours at $z=1$ is due to the assumed EBL evolution (see text).

VHEGRs emitted with energy $E$ from an object located at $z$ is

$$
\tau_{E}(E, z) \equiv \int_{0}^{z} \frac{c d z^{\prime}}{D_{\mathrm{pp}}\left[E\left(1+z^{\prime}\right) /(1+z), z^{\prime}\right] H\left(z^{\prime}\right)\left(1+z^{\prime}\right)} \propto E,
$$

(in which $H(z)$ is the Hubble factor), is shown in the top panel of Figure $1{ }^{6}$ From this it is evident that above $100 \mathrm{GeV}$ the Universe is optically thick to sources at $z>1$ (cf. Franceschini et al. 2008). A related, and perhaps more appropriate measure of the optical depth is that across a Hubble length, $\tau_{H} \equiv c /\left(D_{\mathrm{pp}} H\right)$, shown in the bottom panel of Figure 1, providing a sense of how opaque the Universe is as a function of redshift. In all cases it is clear that at $E \gtrsim 100 \mathrm{GeV}$ photons will pair-produce on the EBL for $z \lesssim 10$. In the future this may not be the case, since for sufficiently small $z, \tau_{H}$ decreases with decreasing $z$ due to the dramatic decrease in the EBL photon density associated with both the Hubble expansion and the slowing of star formation.

\subsection{Temperature of the Ultra-Relativistic Pair Beam}

The pairs produced by the $\mathrm{TeV}$ gamma rays are ultrarelativistic, with typical Lorentz factors of $E / 2 m_{e} c^{2} \simeq$ $10^{6}(E / \mathrm{TeV})$. They also necessarily constitute a cold, highly anisotropic, dilute beam propagating through the IGM. This follows immediately from the intergalactic distances traversed by the gamma rays and the comparatively small EBL photon energies (as seen in the IGM frame). Here we estimate the properties of this plasma beam.

sate for the lost flux until $z=1$. See, for example, Figure 3 from Franceschini et al. (2008).

${ }^{6}$ Note that the optical depth experienced by a photon that is observed to have energy $E^{\prime}$ is given by $\tau_{E}\left[E^{\prime}(1+z), z\right]$. 

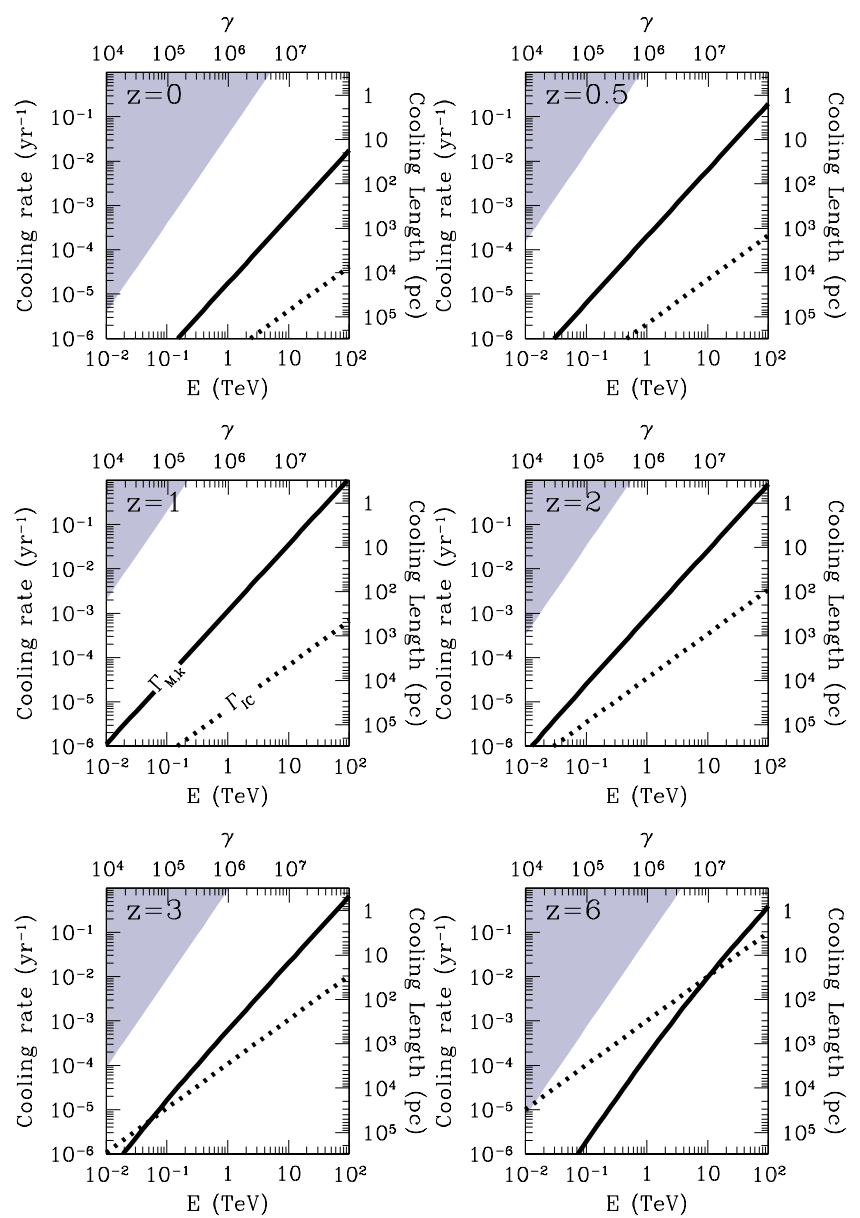

Figure 2. Initial pair beam cooling rates due to the kinetic oblique instability (thick solid) and inverse Compton scattering (dotted) as a function of VHEGR energy $(E)$ at a number of redshifts $(z)$. In all cases $1+\delta=1$, corresponding to a mean-density region, and the isotropic-equivalent luminosity of the source at energy $E, E L_{E}$, is $10^{45} \mathrm{erg} \mathrm{s}^{-1}$, similar to the brightest TeV blazars seen from Earth. Finally, we list the initial pair Lorentz factor, $\gamma$, and cooling lengthscale along the top and right axes, respectively.

The momentum dispersion of the resulting pairs is set by the gamma-ray spectrum, geometry of the TeV source, distribution of the EBL photons, and heating due to pair production. Of these, only the last plays a significant role in setting the transverse momentum dispersion. ${ }^{7}$ The center-of-mass frame, i.e., the "beam frame", momentum dispersion resulting from pair production is roughly $m_{e} c$. This results in an IGM-frame transverse momentum dispersion of $p_{\perp} / p_{\|} \simeq 10^{-6}(E / \mathrm{TeV})^{-1}$. With $p_{\perp}, m_{e} c \ll p_{\|}$, the temperature ${ }^{8}$ associated with this

\footnotetext{
7 The contribution to the transverse momentum dispersion from the finite source size, due to the slightly different orientations of photons from opposing sides of the $\mathrm{TeV}$ emission region of size $\ell$, is $p_{\perp} / p_{\|} \lesssim \ell / 2 D_{\mathrm{pp}}$. With $\ell \simeq$ $10^{14} \mathrm{~cm}$, implied by the X-ray variability timescales (Tramacere et al. 2009; Abdo et al. 2010d), this gives $p_{\perp} / p_{\|} \lesssim 5 \times 10^{-13}[(1+z) / 2]^{\zeta}(E / \mathrm{TeV})^{2}$. Similarly, since the creation of pairs is dominated by EBL photons near the pair-production threshold, the typical energy of the relevant EBL photons is roughly $4 m_{e}^{2} c^{4} / E$ (i.e., twice the threshold value for transverse EBL photons), and thus $p_{\perp} / p_{\|} \simeq 4 m_{e}^{2} c^{4} / E^{2} \simeq 1 \times 10^{-12}(E / \mathrm{TeV})^{-1}$.

${ }^{8}$ The temperature of an anisotropic particle distribution is inherently ill defined. Here we identify a temperature with the momentum dispersion of the beam using the drifting Maxwell-Jüttner distribution employed by Bret et al. (2010a) (eq. 1 therein).
}

transverse momentum dispersion is

$$
\frac{k T_{b}}{m_{e} c^{2}} \simeq \frac{p_{\|}}{2 m_{e} c^{2}}\left(\frac{p_{\perp}}{p_{\|}}\right)^{2} \simeq 5 \times 10^{-7}\left(\frac{E}{\mathrm{TeV}}\right)^{-1} .
$$

Since the transverse momentum dispersion of the pairs is much smaller than that associated with the bulk motion of the beam (i.e., since $p_{\perp} / p_{\|} \ll 1$ ), we may safely assume that the beam is transversely kinematically cold.

\subsection{Density of the Ultra-Relativistic Pair Beam in General}

The density of the pair beam at a given point within the IGM is set by the rate at which pairs are produced, duration of the $\mathrm{TeV}$ emission, advection of the pairs through the IGM, and the processes by which they lose their kinetic energy. That is, the evolution of the density of pairs per unit Lorentz factor, $n_{\gamma}$, is governed by the Boltzmann equation:

$$
\frac{\partial n_{\gamma}}{\partial t}+\frac{c}{r^{2}} \frac{\partial r^{2} n_{\gamma}}{\partial r}+\dot{\gamma} \frac{\partial n_{\gamma}}{\partial \gamma}=\dot{n}_{\gamma}
$$

where the left-hand side assumes all the pairs are moving away from the $\mathrm{TeV}$ source relativistically $\left(v^{r}=c\right.$ and $\left.p^{r}=\gamma m_{e} c\right)$, and the right-hand side corresponds to pair production. Generally, we may neglect advection, which alters $n_{\gamma}$ over timescales of $D_{\mathrm{pp}} / c$, much longer than any relevant timescale of interest here (i.e., $\left(c / r^{2}\right) \partial r^{2} n_{\gamma} / \partial r$ may be neglected). Furthermore, for most of the potential sources we will consider (primarily TeV blazars) we will assume that the duration of the TeV emission is sufficiently long that $n_{\gamma}$ reaches a steady state (i.e., $\left.\partial n_{\gamma} / \partial t=0\right)$. In this case, we have $\dot{\gamma} \partial n_{\gamma} / \partial \gamma \simeq \dot{n}_{\gamma}$.

Making further progress requires us to define the spectrum of the pairs, which itself depends upon the spectrum of the gamma rays and the energy dependence of the cooling processes. Nevertheless, we may obtain an estimate of the beam density in the vicinity of a given Lorentz factor, $n_{b} \simeq \gamma n_{\gamma}$, by setting $\partial n_{\gamma} / \partial \gamma \simeq-n_{\gamma} / \gamma=-n_{b} / \gamma^{2}$.

The source term is given by twice (since each gamma-ray produces two leptons) the rate at which high-energy gamma rays with energy $E \simeq 2 \gamma m_{e} c^{2}$ annihilate within the region of interest, i.e., $\gamma \dot{n}_{\gamma}=2(E d N / d E) / D_{\mathrm{pp}}=2 F_{E} / D_{\mathrm{pp}}$, where $N$ is the gamma-ray number flux, with units of photons $\mathrm{cm}^{-2} \mathrm{~s}^{-1}$. Thus, upon defining a cooling rate $\Gamma \equiv-\dot{\gamma} / \gamma$, we have

$$
n_{b} \simeq \frac{2 F_{E}}{D_{\mathrm{pp}} \Gamma},
$$

i.e., the density of pairs of a given energy is determined by balancing cooling and pair creation.

Generally, $\Gamma$ is a function of energy and beam density, as well as external factors (e.g., seed photon density, IGM density, etc.). Thus this gives a non-linear algebraic equation to solve for $n_{b}$, the particulars of which depend upon the various mechanisms responsible for extracting the bulk energy of the beam. In practice, given expressions for $\Gamma$, associated with the processes discussed in following section, we solve Equation (5) numerically to obtain $n_{b}\left(E, F_{E}, z\right)$.

Which mechanism dominates the cooling of the beam depends upon a variety of environmental factors and the properties of the pair beam itself. Nevertheless, inverse-Compton cooling via the cosmic microwave background (CMB) provides a convenient lower limit upon $\Gamma$, and thus an upper limit upon $n_{b}$. This is a function of $z$ and $\gamma$ alone, given by

$$
\Gamma_{\mathrm{IC}}=\frac{4 \sigma_{\mathrm{T}} u_{\mathrm{CMB}}}{3 m_{e} c} \gamma \simeq 1.4 \times 10^{-20}(1+z)^{4} \gamma \mathrm{s}^{-1},
$$


where $\sigma_{\mathrm{T}}$ denotes the Thompson cross section. The strong redshift dependence arises from the rapid increase in the CMB energy density with $z\left(u_{\mathrm{CMB}} \propto(1+z)^{4}\right)$. Furthermore, since it is $\propto \gamma$, inverse-Compton cooling is substantially more efficient at higher energies. The associated cooling rate is shown as a function of $E$ for a number of redshifts in Figure 2.

When we set $\Gamma \simeq \Gamma_{\text {IC }}$, we obtain the following upper limit upon the beam density:

$$
\begin{aligned}
n_{b} & \simeq \frac{2 F_{E}}{D_{\mathrm{pp}} \Gamma_{\mathrm{IC}}} \simeq \frac{L_{E}}{2 \pi D_{\mathrm{pp}}^{3} \Gamma_{\mathrm{IC}}} \\
& \simeq 3.7 \times 10^{-22}\left(\frac{1+z}{2}\right)^{3 \zeta-4}\left(\frac{E L_{E}}{10^{45} \mathrm{erg} \mathrm{s}^{-1}}\right)\left(\frac{E}{\mathrm{TeV}}\right) \mathrm{cm}^{-3},
\end{aligned}
$$

Where we have defined $L_{E}$ to be the isotropic-equivalent luminosity (per unit energy) of a source located a distance $D_{\mathrm{pp}}$ from the region in question. Setting $E L_{E}$ to a typical value $\left(10^{45} \mathrm{erg} \mathrm{s}^{-1}\right)$ gives an idea of the typical pair-beam densities. Note that despite the large blazar luminosities we consider, the associated beams are exceedingly dilute, a point that is of critical importance in the following section. Since $\Gamma_{\mathrm{IC}}$ is independent of $n_{b}$, this has no implication for inverse-Compton cooling itself.

\section{COOLING ULTRA-RELATIVISTIC PAIR BEAMS VIA PLASMA BEAM INSTABILITIES}

Plasma beams are notoriously unstable, with the instabilities driven by the anisotropy of the lepton distribution function. Here we consider the implications of these instabilities upon the ultimate fate of the kinetic energy in the TeV-blazar driven pair beams.

\subsection{A Fundamental Limit Upon Plasma Cooling Rates}

For collective phenomena to be relevant, it is necessary for many pairs to be present within each wavelength of the unstably growing modes. As we shall see, the relevant scale for the beam plasma instabilities we describe below is the plasma skin depth of the IGM,

$$
\lambda_{P} \equiv \frac{2 \pi c}{\omega_{P}}=\sqrt{\frac{\pi m_{e} c^{2}}{e^{2} n_{\mathrm{IGM}}}}=2.3 \times 10^{-9}(1+\delta)^{-1 / 2}(1+z)^{-3 / 2} \mathrm{pc},
$$

where $\omega_{P} \equiv \sqrt{4 \pi e^{2} n_{\mathrm{IGM}} / m_{e}}$ is the IGM plasma frequency and $n_{\mathrm{IGM}}=2.2 \times 10^{-7}(1+\delta)(1+z)^{3} \mathrm{~cm}^{-3}$ is the IGM free-electron number density. Generally, the growing mode must be uniform on scales considerably larger than $\lambda_{P}$, both longitudinally and transversely (otherwise it is not well-represented as a single Fourier component), and thus the volume in which many particles must be present is much larger than that defined by a sphere of diameter $\lambda_{P}$. Nevertheless, this gives us a conservative constraint, i.e., we require

$$
\frac{\pi}{6} \lambda_{P}^{3} n_{b} \gg 1
$$

With Equation (5) this gives a maximum plasma cooling rate:

$$
\Gamma_{\text {plasma }} \ll \frac{\pi}{3} \frac{F_{E}}{D_{\mathrm{pp}}} \lambda_{P}^{3} .
$$

Plasma processes with cooling rates that exceed this limit necessarily saturate near this cooling rate. Such a super-critical process can potentially operate only until the beam density is

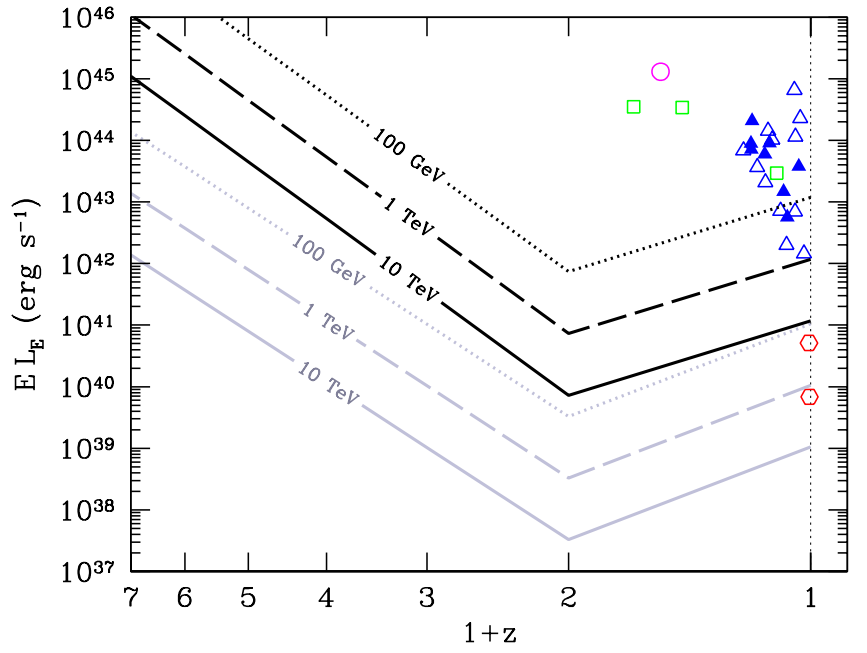

Figure 3. Limiting isotropic-equivalent luminosities as a function of redshift for various gamma-ray energies for $1+\delta=1$ region of the IGM. Grey lines show the limit defining the applicability of the plasma prescription, below which less than a single beam lepton is found in a volume $\pi \lambda_{p}^{3} / 6$. Below these collective phenomena are unimportant. Black lines show the luminosity at which linear plasma cooling rate begins to dominate inverse-Compton cooling. Both limits are sensitive functions of the IGM density, scaling as $(1+\delta)^{3 / 2}$ and $(1+\delta)^{1 / 2}$, respectively. Thus in low/high density regions these limits become moderately more/less permissive. For reference, the sources listed in Table 1 are also plotted (at $E=1 \mathrm{TeV}$ ), with $\mathrm{HBL}$, IBL, radio galaxies, and quasars shown by the the blue triangles, green squares, red hexagons and magenta circles, respectively. Filled points indicate sources that have been used to estimate the IGMF (see Section 4). The only objects that fail to meet the plasma-cooling luminosity criterion are the radio galaxies M87 and Cen A, both of which are detectable only due to their close proximity.

driven below the value at which pairs can support collective phenomena, at which point they necessarily quench. However, after plasma cooling ceases, the plasma beam density rises again (since $\Gamma_{\mathrm{IC}}$ is always less than $\Gamma_{\text {plasma }}$ in practice), and thus the super-critical plasma cooling may resume. Since the efficiency of the plasma cooling decreases smoothly to zero at the critical density, this sequence stabilizes near $\Gamma_{\text {plasma. }}$. The associated excluded region is shown by the grey region in the upper-left corner of Figure 2.

The upper limit upon $n_{b}$ obtained in Equation (7) implies an analogous limit upon the relevant source isotropic-equivalent luminosities. Again, this arises from requiring a sufficient number of beam pairs to be present to support plasma processes. Since the beam density is linearly dependent upon the source luminosity, this gives a constraint upon the latter:

$$
\begin{aligned}
E L_{E} & \gg 12 E \frac{D_{\mathrm{pp}}^{3} \Gamma_{\mathrm{IC}}}{\lambda_{P}^{3}} \\
& =3.3 \times 10^{38}(1+\delta)^{3 / 2}\left(\frac{1+z}{2}\right)^{8.5-3 \zeta}\left(\frac{E}{\mathrm{TeV}}\right)^{-1},
\end{aligned}
$$

effectively defining a luminosity cut-off, below which collective plasma phenomena may be ignored. This limiting luminosity shown as a function redshift by the grey lines in Figure 3 for various gamma-ray energies. While the luminosity limit does depend upon $z$, it is clear that all of the observed TeV blazars (listed in Table 1) are sufficiently luminous for their resulting pair beams to support collective phenomena at the redshifts of interest for active galactic nuclei (AGNs). The only source to fall marginally below the limiting luminosity at $1 \mathrm{TeV}$ is the ra- 
dio galaxy Cen A (the lower-most point in Figure 3), the closest and dimmest object in Table 1.

\subsection{Cold Plasma Beam Instabilities}

Within astrophysical contexts there are at least two well known beam instabilities: two-stream and Weibel, the latter having been suggested as a mechanism for magnetizing strong shocks (Medvedev \& Loeb 1999), and both implicated in the coupling at collisionless shocks (Spitkovsky 2008). These are, however, simply different limiting examples of the same underlying filamentary instability, for which the maximum growth rate exceeds either - the so-called "oblique" mode, which we discuss below (Bret et al. 2004, 2005a; Lemoine \& Pelletier 2010). Note that since the pair beam is neutral, it contains its own return current and thus beam instabilities that arise due to the electron return currents within the background plasma (e.g., the Bell and Buneman instabilities, see Bret 2009) are not relevant.

Here we discuss the nature of these instabilities, and their growth rates in the cold-plasma limit (i.e., mono-energetic beams). While the low-temperature approximation is unlikely to be applicable in practice for the beams of interest here, it provides a convenient limit in which to present the relevant processes within their broader context. For a similar reason, and because we have not found analogous derivations elsewhere in the astrophysical literature, we present the ultra-relativistic pair beam instability growth rates for the two-stream and Weibel instabilities in Appendixes A.1 and A.2, only summarizing the results here. We defer a discussion of the more directly relevant warm-plasma oblique instability to the following section. Generally, we find that the plasma instabilities are capable of dominating inverse Compton cooling as a means to dissipate the bulk kinetic energy of the pair beams from TeV blazars.

The pair two-stream instability arises due to the interaction of the anisotropic electron and positron distribution functions with the comoving background electrostatic wave (i.e., $\mathbf{k} \| \mathbf{p}$, where $\mathbf{k}$ is the electromagnetic mode wavevector and $\mathbf{p}$ is the beam momentum) with wavelength $\sim \lambda_{P}$ for $n_{b} / n_{\mathrm{IGM}} \ll 1$, which is generally the case of interest here. The associated cooling rate ${ }^{9}$ in the cold-plasma limit is

$$
\Gamma_{\mathrm{TS}}=\sqrt{\frac{12 \pi e^{2} n_{\mathrm{IGM}}}{m_{e}}}\left(\frac{n_{b}}{2 n_{\mathrm{IGM}}}\right)^{1 / 3} \frac{1}{\gamma},
$$

which depends only weakly upon the IGM density and the pair beam density, though decreases rapidly as $\gamma$ becomes large.

The Weibel instability is associated with coupling to a secularly growing, anharmonic transverse magnetic perturbation (i.e., $\mathbf{k} \perp \mathbf{p}$ ). The most rapidly growing wavelength is again that associated with the background plasma skin depth, $\sim \lambda_{P}$, with associated cooling rate in the cold-plasma limit

$$
\Gamma_{\mathrm{W}}=\sqrt{\frac{16 \pi e^{2} n_{b}}{m_{e} \gamma}},
$$

which depends only upon the pair beam density and the pair Lorentz factor. At large $\gamma$ this is suppressed more weakly than the two-stream instability, though is a moderately stronger function of $n_{b}$.

\footnotetext{
${ }^{9}$ Note that since we are interested in the rate at which energy is lost, these are necessarily twice the instability "growth" rates, defined by the e-folding time of the electromagnetic wave amplitudes.
}

The computation of the growth rates of the two-stream and Weibel instabilities are greatly simplified by the particular geometries of the coupled electromagnetic waves and beam momenta. However, a generalized oblique treatment has shown the presence of continuum of unstable modes (Bret et al. 2004, 2005a; Bret 2009; Bret et al. 2010b; Lemoine \& Pelletier 2010), characterized by the orientation of their wave-vector relative to the bulk beam velocity. Of these neither the twostream nor Weibel are generally the most unstable. Rather, generically the most robust and fastest growing mode occurs at oblique wave-vectors, and thus referred to as the oblique instability by Bret et al. (2010b). The cold-plasma cooling rate of this maximally-growing mode is

$$
\Gamma_{\mathrm{M}} \simeq \sqrt{\frac{12 \pi e^{2} n_{\mathrm{IGM}}}{m_{e}}}\left(\frac{n_{b}}{2 \gamma n_{\mathrm{IGM}}}\right)^{1 / 3}=\Gamma_{\mathrm{TS}} \gamma^{2 / 3} .
$$

This can be much larger than the two-stream and Weibel growth rates when $n_{b} / n_{\text {IGM }} \ll 1$ and $\gamma \gg 1$.

\subsection{Warm Plasma Beam Instabilities}

The cold-plasma oblique instability cooling rates dominate inverse-Compton cooling by orders of magnitude over the region of interest. However, at the very dilute beam densities of relevance here, the cold-plasma approximation requires exceedingly small beam temperatures. Above an IGM-frame temperature of roughly

$$
\begin{aligned}
\frac{k T_{\text {crit }}}{m_{e} c^{2}} & \simeq \frac{3}{2^{10 / 3}}\left(\frac{n_{b}}{n_{\mathrm{IGM}}}\right)^{2 / 3} \gamma^{1 / 3} \\
& \simeq 1.0 \times 10^{-9}(1+\delta)^{-2 / 3}\left(\frac{1+z}{2}\right)^{2 \zeta-14 / 3} \\
& \times\left(\frac{E L_{E}}{10^{45} \mathrm{erg} \mathrm{s}^{-1}}\right)^{2 / 3}\left(\frac{E}{\mathrm{TeV}}\right),
\end{aligned}
$$

pairs can traverse many wavelengths of the unstable modes over the cold-instability growth timescale, a situation commonly referred to as the kinetic regime. As a consequence, significant phase mixing can occur, substantially reducing the effective growth rate (Bret et al. 2010a).

The way in which finite beam temperatures limit the growth rate depends sensitively upon the nature of the velocity dispersion and the modes of interest (see, e.g., Bret et al. 2005b). For example, the two-stream instability, associated with wave vectors parallel to the beam, enters the strongly suppressed "quasilinear" regime when the parallel momentum dispersion is large, though is insensitive to even large transverse dispersions. Conversely, the Weibel instability, associated with wave vectors orthogonal to the beam, is sensitive to even small transverse velocity dispersions but unaffected by large parallel velocity dispersions. This is simply because a given mode can tolerate large velocity dispersions within but not across the phase fronts of the unstable electromagnetic modes. For the situation of interest here, dilute beams and cool IGM (i.e., $k T_{\mathrm{IGM}} / m_{e} c^{2} \ll 1$ ), the oblique modes are nearly transverse, and thus sensitive primarily to large transverse velocity dispersions.

Nevertheless, even for the small temperatures we have inferred for the pair beams, we find ourselves in the kinetic regime. In this case the oblique instability cooling rate has been numerically measured to be

$$
\Gamma_{\mathrm{M}, \mathrm{k}} \simeq 0.4 \frac{m_{e} c^{2}}{k T_{b}} \frac{n_{b}}{n_{\mathrm{IGM}}} \omega_{P} \simeq 0.4 \gamma \sqrt{\frac{4 \pi e^{2} n_{b}^{2}}{m_{e} n_{\mathrm{IGM}}}},
$$


where we have set the beam temperature in the beam frame to $m_{e} c^{2} / k$, and thus $k T_{b}=m_{e} c^{2} / \gamma$ (Bret et al. 2010a). Both the cold and hot growth rates have been verified explicitly using particle-in-cell (PIC) simulations, though for somewhat less dilute beams than those we discuss here (Bret et al. 2010b).

When the kinetic oblique mode dominates the beam cooling the beam density is given by

$$
\begin{aligned}
n_{b} \simeq 2.1 \times 10^{-24} & (1+\delta)^{1 / 4}\left(\frac{1+z}{2}\right)^{(6 \zeta+3) / 4} \\
& \times\left(\frac{E L_{E}}{10^{45} \mathrm{erg} \mathrm{s}^{-1}}\right)^{1 / 2}\left(\frac{E}{\mathrm{TeV}}\right)^{1 / 2} \mathrm{~cm}^{-3} .
\end{aligned}
$$

The associated cooling rate is then

$$
\begin{aligned}
\Gamma_{\mathrm{M}, \mathrm{k}} \simeq 3.6 \times 10^{-11} & (1+\delta)^{-1 / 4}\left(\frac{1+z}{2}\right)^{(6 \zeta-3) / 4} \\
& \times\left(\frac{E L_{E}}{10^{45} \mathrm{erg} \mathrm{s}^{-1}}\right)^{1 / 2}\left(\frac{E}{\mathrm{TeV}}\right)^{3 / 2} \mathrm{~s}^{-1} .
\end{aligned}
$$

This is a stronger function of gamma-ray energy than inverseCompton cooling, implying that it will eventually dominate at sufficiently high energies, assuming a flat $\mathrm{TeV}$ spectrum. In addition it is a very weak function of $\delta$, being only marginally faster in lower-density regions, and thus the cooling of the pairs is largely independent of the properties of the background IGM.

The rates obtained by numerically solving Equation (5) for $n_{b}$, with $\Gamma=\Gamma_{\mathrm{IC}}+\Gamma_{\mathrm{M}, \mathrm{k}}$, are shown for a number of redshifts in Figure 2. For the luminosity shown $\left(E L_{E}=10^{45} \mathrm{erg} \mathrm{s}^{-1}\right.$, typical of the bright TeV blazars) at $z \lesssim 4$ plasma cooling dominates inverse Compton above a TeV. In the present epoch, $\Gamma_{\mathrm{M}, \mathrm{k}}$ is roughly two orders of magnitude larger than $\Gamma_{\mathrm{IC}}$ for bright $\mathrm{TeV}$ blazars.

The luminosity dependence of $\Gamma_{\mathrm{M}, \mathrm{k}}$ implies a luminosity limit below which inverse Compton does dominate the linear evolution of the pair beam,

$$
E L_{E} \gtrsim 7.4 \times 10^{40}(1+\delta)^{1 / 2}\left(\frac{1+z}{2}\right)^{9.5-3 \zeta}\left(\frac{E}{\mathrm{TeV}}\right)^{-1}
$$

(note that at this luminosity $\Gamma=\Gamma_{\mathrm{IC}}+\Gamma_{\mathrm{M}, \mathrm{k}}=2 \Gamma_{\mathrm{M}, \mathrm{k}}$, and thus $n_{b}$ is half the value shown in Equation (17)). This limit is shown as a function of redshift for a number of different energies by the black lines in Figure 3. Note that these lie above the corresponding limits associated with the applicability of the plasma prescription, suggesting that in practice the beam does support collective phenomena. The critical luminosity ranges from $\sim 10^{40} \mathrm{erg} \mathrm{s}^{-1}$ to $10^{45} \mathrm{erg} \mathrm{s}^{-1}$, depending upon redshift and energy of interest. It also depends upon the over-density as roughly $\propto(1+\delta)^{1 / 2}$, and thus at low densities the critical luminosity is moderately smaller. The only two sources in Table 1 which fall below the plasma-cooling luminosity limit are the radio galaxies M87 and Cen A, both of which are detectable only as a result of their close proximity. At $1 \mathrm{TeV}$, all but a handful of the remaining sources lie more than an order of magnitude above this limit, and in the case of the two sources that dominate the $\mathrm{TeV}$ flux at Earth, more than two orders of magnitude above this limit.

\subsection{Intuitive Picture of the two-stream, Weibel, and Oblique Instabilities}

Given the technical nature of our discussion above, it is useful to have a qualitative understanding of these instabilities. We caution, however, that intuitive pictures of plasma processes frequently fail to capture all the relevant physics. Hence, generalization of these intuitive pictures beyond their limited range of applicability is potentially misleading. This is explicitly illustrated by our examples here: all of the instabilities discussed in this work belong to the same family, i.e., instabilities that arise from interpenetrating plasmas, but the underlying qualitative pictures for each differ substantially.

We begin with the Weibel (or filamentation) instability (Weibel 1959), for which a mechanical viewpoint (an initial magnetic perturbation deflects particles into opposing current streams that reinforce the perturbed field) can be found in Medvedev \& Loeb (1999), to which we refer the interested reader. Here we present picture that although having the virtue of being simpler is not entirely correct: because like currents attract, small-scale current perturbations arising out of the fluctuations within the interpenetrating plasmas will coalesce preferentially to produce increasingly larger-scale currents. These induce stronger magnetic fields, and thus larger attractive Lorentz forces between neighboring currents; a positive feedback loop develops leading to instability. This process continues until the associated magnetic field strengths become sufficiently large to disrupt the currents (in the case of equal density beams) or until the transverse velocity of the constituent particles is large enough to efficiently migrate between current structures on the linear growth timescale, i.e., enter the kinetic regime.

We should note that the aggregation of currents in the Weibel instability does not rely upon oscillatory waves. ${ }^{10}$ Hence, there is no oscillatory component to this instability - it is a purely growing mode. In contrast, the two-stream instability is an overstable mode, where the oscillatory components are Langmuir (or plasma) waves with a wavevector parallel to the beam velocity. These are longitudinal waves, associated with local charge oscillations, and are completely described by a propagating perturbation in the electrical potential. As particles in the beam traverse Langmuir waves in the background plasma, they experience successive periods of acceleration and deceleration, with electrons (positrons) collecting in minima (maxima) of the electric potential, where the particle speeds are at their smallest. ${ }^{11}$ This charge-separated bunching of the beam plasma enhances the background electric perturbation, potentially growing the background Langmuir wave.

The bunching within the beam is simply an excitation of Langmuir waves within the beam plasma itself. Thus the growth of the charge perturbations in the background and beam plasmas corresponds to the resonant coupling between Langmuir waves in the background and beam. When the beam density is much less than the background density, as is the case here, background and beam Langmuir waves only overlap in frequency, and therefore satisfy the conditions for resonance, when the wavevector of the latter is parallel to the beam veloc-

\footnotetext{
10 The linear analysis of the Weibel instability assumes wave-like disturbances in the plasma. However, there is no associated restoring force to these waves and, hence, they do not oscillate.

${ }^{11}$ One tempting aspect is to think of these particles as almost massless and hence imagining that these electric fields are quickly shorted out. While this is the case in most astrophysical process, we urge the reader to resist this temptation because these instabilities occur on short enough timescales that the mass of these charged particles plays a crucial role in the physics of the instability.
} 
ity (see the discussion above Equation (A9)). This is always satisfied for the family of comoving beam Langmuir waves (i.e., waves which in the beam frame move counter to the background plasma). However, of particular importance for the two-stream instability are the counter-propagating beam Langmuir waves (i.e., waves which in the beam frame move parallel to the background plasma). If the beam velocity exceeds the phase velocity of these waves (as seen in the beam frame), the counter-propagating wave will be dragged in the direction of the beam (as seen in the background frame), and therefore has a wavevector which satisfies the resonant condition.

Nevertheless, the counter-propagating Langmuir wave still carries momentum in the direction opposite to the beam. Thus, as the counter-propagating wave grows, the momentum, and therefore energy, of the beam-wave system necessarily decreases. This implies that the counter-propagating Langmuir wave is also a negative energy mode as seen in the background frame. As a consequence, the resonant coupling can transfer energy to the positive-energy background wave from the negative-energy beam wave, while growing the amplitudes of both, and thereby leading to instability. Note that if the phase velocity of the counter-propagating Langmuir wave is larger than the beam velocity, it no longer is dragged in the direction of the beam and no longer satisfies the necessary resonant condition. For a distribution of particles, this constraint upon the velocities within the beam corresponds to the familiar Penrose criterion (Sturrock 1994; Boyd \& Sanderson 2003), and is satisfied in the pair beams resulting from VHEGRs.

The oblique instability encompasses the two-stream and Weibel instabilities, though the most unstable mode is most similar to the former in that the intuitive picture focus solely on the electrostatic forces, ignoring electromagnetic forces. ${ }^{12}$ In practice, this is a relatively good approximation and can be used to calculate the growth of these modes in idealized situations (e.g. Nakar et al. 2011; Bret et al. 2004). The qualitative picture proceeds similarly to that for the two-stream instability described above, with the minor modification that the perturbing background Langmuir waves now move at an angle $\theta$ relative to the relativistic beam. As a consequence, the resonant Langmuir waves have a phase velocity such that $v_{k} \approx c \cos \theta$. From the simple intuitive picture above, if $\theta$ is selected such that the projected beam velocity is slightly faster than the nearly resonant Langmuir wave, an instability develops.

Finally, to understand why the growth rates between the twostream instability above and the oblique instability differ, it is useful to make the approximation that the electric fields generated are in the direction of the k-vector. In the two-stream case, the electric field must slow down (or speed up) particles. This gets progressively harder for more relativistic particles. In the oblique case, the electric field deflects the particles, changing their projected velocity. While this is also more difficult for more relativistic particles, this is not nearly as hard as changing the particles parallel (along the beam) velocity. Hence, the oblique instability more easily drives charge density enhancements (and therefore instabilities) at large $\theta$, i.e., easier deflection, than the two-stream instability.

\subsection{Non-Linear Saturation}

We have thus far only treated the linear development of the relativistic two-stream, Weibel, and oblique instabilities. How-

\footnotetext{
12 The picture we present here for the oblique instability is discussed in Nakar et al. (2011).
}

ever, the impact pair beams have upon the IGM, gamma-ray cascade emission, and measures of the IGMF will ultimately depend upon their nonlinear development. To address this, however, we are presently forced to appeal to analytical and numerical calculations of systems in somewhat different (and less extreme) parameter regimes.

Motivated by the applicability of the Weibel instability in the context of GRBs, the nonlinear saturation of the relativistic Weibel instability for equal density plasma beams is well understood analytically and numerically. Initially, the Weibel instability rapidly grows until the energy density of the generated magnetic field becomes of order the kinetic energy of the two beams (Silva et al. 2003; Frederiksen et al. 2004; Chang et al. 2008). Analytically, Davidson et al. (1972) argued that the Weibel instability would saturate when the generated magnetic fields become so large that the Larmor radius of the beam particles is of order the skin depth, i.e., when the energy of generated magnetic fields is equal to the kinetic energy of two equal density relativistic beams (see also Medvedev \& Loeb 1999). The particles rapidly isotropize with a Maxwellian distribution (Spitkovsky 2008), i.e., heat, and the magnetic energy then rapidly decays within an order of a few tens of skin depths (Chang et al. 2008). Hence for two equal density, relativistic, interpenetrating beams, the Weibel instability converts anisotropic kinetic energy into heat. However, as we have already noted, for the pair beams of interest here the Weibel instability is completely suppressed for tiny transverse beam temperatures. Hence, while this instability may initially operate, it may quickly become suppressed if it results in significant transverse heating of the beam.

Unlike the Weibel instability, the two-stream and kinetic oblique instabilities continue to operate, though more slowly than implied by their cold-plasma limits, in the presence of substantial beam temperatures. Due to the geometry of the coupled modes, the oblique instability is primarily sensitive to transverse beam-velocity dispersions, though shares the resistance to beam heating with its two-stream cousin (Bret et al. 2005b; Bret 2009; Bret et al. 2010b; Lemoine \& Pelletier 2010). Unlike the two-stream instability, the oblique instability in the parameter range of interest here is also largely insensitive to longitudinal heating. Thus, generally, it appears that the oblique instability is substantially more robust than its more commonly discussed brethren.

What is less clear a priori is if these instabilities primarily heat the beam or primarily heat the background plasma. Here we appeal to the numerical simulations of Bret et al. (2010b), where a mildly relativistic beam $(\gamma=3)$ penetrating into a hot, dense background plasma (beam-to-background density ratio of 0.1 ) was studied. In these the oblique instability resulted in a significant fraction $(\sim 20 \%)$ of the beam energy heating the background plasma before the heating of the beam suppressed the oblique instability in favor of the two-stream instability. The relative effectiveness with which the beam heats the background plasma is due to the efficiency with which the longitudinal electrostatic modes are dissipated in the background plasma; unlike electromagnetic modes (e.g., those generated by the Weibel instability), electrostatic modes are rapidly dissipated via Landau damping.

We note that Bret et al. (2010b) found that the heating of the beam by the oblique instability eventually led to its suppression, allowing the two-stream instability to grow, continuing the dissipation of the beam kinetic energy. As a result, in their simulations a total of $30 \%$ of the energy was deposited into the background plasma via a combination of the oblique and two- 
stream instabilities, i.e., an additional $10 \%$ of the beam energy was thermalized via the two-stream instability during and after the suppression of the oblique instability.

In our case, we expect that much more beam energy (more than the $20 \%$, and possibly up to $\sim 100 \%$ ) will be deposited into the background IGM because we are much deeper into the regime in which the oblique instability dominates, i.e., $\gamma \gg 1$ and $n_{b} / n_{\mathrm{IGM}} \ll 1$. In particular, for the case of interest here, $\gamma \sim 10^{6}$ and

$$
\begin{aligned}
\frac{n_{b}}{n_{\mathrm{IGM}}} \simeq 1.3 \times & 10^{-18}(1+\delta)^{-3 / 4}\left(\frac{1+z}{2}\right)^{(6 \zeta-9) / 4} \\
& \times\left(\frac{E L_{E}}{10^{45} \mathrm{erg} \mathrm{s}^{-1}}\right)^{1 / 2}\left(\frac{E}{\mathrm{TeV}}\right)^{1 / 2} \mathrm{~cm}^{-3} .
\end{aligned}
$$

The extremely large Lorentz factor and tiny density ratio make it computationally prohibitive to assess the beam evolution with numerical PIC directly. Nevertheless, because we find ourselves in a regime in which the oblique instability is much more strongly dominant than that simulated in Bret et al. (2010b), we expect the linear growth of the kinetic oblique instability to continue for much longer before the beam changes character and moves out of the oblique-dominated regime potentially once $\sim 100 \%$ of the beam energy has been dissipated. However, this remains to be studied in future work.

The effect of nonlinear processes might also effect the evolution of the linear instability. For instance, Lesch \& Schlickeiser (1987) argued that for the relativistic electrostatic twostream instability, nonlinear coupling to daughter modes arrest the growth of the linearly unstable mode at a very low mode energy. Hence, they claim that the electrostatic two-stream instability can only bleed energy from the beam at a slow rate.

Utilizing the order of magnitude estimates in Lesch \& Schlickeiser (1987) or the expression for nonlinear Landau damping in Melrose (1980), we have found that damping of the pair beam via the relativistic two-stream instability could be highly suppressed. The oblique instability may be similarly suppressed, however, this effect is much more marginal due to the instabilities much larger growth rate. Nevertheless, determining its behavior for the parameters relevant here is an important unanswered question that is left for future work. For the purposes of this paper, we will rely upon the intuition developed from numerical studies of the oblique instability and argue that the beam ends up heating the IGM primarily.

In what follows, we will presume the nonlinear evolution of the "oblique" or related plasma instabilities lead to the heating of the background IGM, i.e., beam cooling. However, we note that the beam itself may be the primary recipient of this kinetic energy, i.e., beam disruption. Most of our results depend critically on beam cooling and not beam disruption. This includes the effect of blazar heating on the IGM temperaturedensity relation studied in Paper II, the effects on structure formation studied in Paper III, the excellent reproduction of the statistical properties of the high-redshift Ly $\alpha$ forest found in Puchwein et al. (2011), and the implications on the EGRB and the redshift evolution of $\mathrm{TeV}$ blazars studied in Section 5. However, our conclusions on the inapplicability of IGMF constraints determined from the non-observation of $\mathrm{GeV}$ emission from blazars still remains in the presence of beam disruption. This is because the self-scattering of pairs in the beam would suppress this $\mathrm{GeV}$ emission in similar manner to an IGMF.

\subsection{Suppression of the Oblique Instability by an IGMF}

The beam instabilities we have discussed have been analyzed primarily within the context of unmagnetized plasmas. However, for a variety of theoretical reasons a weak IGMF is not unexpected. For example, a field strength of $10^{-15} \mathrm{G}$ is sufficient to explain the observed $\mathrm{nG}$ galactic fields via compression and winding alone. Here we consider the implications that an IGMF has for the instability growth rates we have described above.

A strong IGMF causes the ultra-relativistic pairs to gyrate, and therefore to isotropize, suppressing the growth of instabilities that feed upon the beam anisotropy (e.g., those we have described above). However, for this to efficiently quench the growth of the plasma beam instabilities, this isotropization must occur on a timescale comparable to the instability growth time, i.e., the Larmor frequency must be comparable to the cooling rate, $\Gamma$. This condition gives a lower-limit upon IGMF strengths sufficient to appreciably suppress the growth of plasma beam instabilities of

$$
B \gtrsim 10^{-12}\left(\frac{\gamma}{10^{6}}\right)\left(\frac{\Gamma}{10^{-4} \mathrm{yr}}\right) \mathrm{G},
$$

considerably larger than those typical of both, primordial formation mechanisms (Widrow 2002) and implied by galactic magnetic field estimates, assuming galactic fields are produced by contraction and winding alone.

Because the VHEGRs emitted by the TeV blazars travel cosmological distances prior to producing pairs, an IGMF capable of suppressing the plasma beam instabilities must necessarily have a volume filling fraction close to unity. In particular, it must permeate the low-density regions, where most of the cooling occurs. However, the Alfvén velocity within those areas is extraordinarily small, roughly

$$
v_{\mathrm{A}} \simeq 4 \times 10^{-3}\left(\frac{B}{10^{-12} \mathrm{G}}\right)(1+\delta)^{-1 / 2} \mathrm{~km} \mathrm{~s}^{-1} .
$$

The IGM sound speed,

$$
c_{s} \simeq 10\left(\frac{T}{10^{4} \mathrm{~K}}\right)^{1 / 2} \mathrm{~km} \mathrm{~s}^{-1},
$$

is considerably larger, implying convection is much more efficient. Nevertheless, even after substantial heating via the thermalization of the TeV blazar emission (Paper II) is taken into account, magnetic fields will have propagated $\lesssim 100 \mathrm{kpc}$ over a Hubble time via convection and much less via diffusion, implying that a pervasive, sufficiently strong magnetic field can not be produced via ejection from galactic dynamos. While galactic winds can produce much faster outflows, $v_{W} \sim 10^{3} \mathrm{~km} \mathrm{~s}^{-1}$, unless they inject a mass comparable to that contained in the low-density regions over a Hubble time, they are rapidly slowed via dissipation at shocks in the IGM, again limiting the spread of galactic fields. Moreover, we note that since the magnetic field must be volume filling to suppress the plasma beam cooling substantially it is insufficient to produce pockets of strong fields, and thus any galactic origin powered by winds requires a nearly complete reprocessing of the low-density regions. However, the ultimate thermalization of such fast, dense winds would raise the IGM temperature to $\sim 10^{8} \mathrm{~K}$, in conflict with the $\operatorname{Ly} \alpha$ forest data and exceeding the entire bolometric output of quasars by at least a factor of two. For these reasons we conclude that a volume-filling strong IGMF would demand a primordial origin. 


\section{IMPLICATIONS FOR IGM MAGNETIC FIELD ESTIMATES}

The existence of plasma processes that can cool the pair beams associated with $\mathrm{TeV}$ blazars has profound consequences for efforts to constrain the IGMF using the spectra of $\mathrm{TeV}$ blazars. Here we describe how the reported IGMF limits have been obtained, the consequences of plasma cooling for these, and potential strategies for overcoming the constraints it imposes.

The general argument made in efforts to constrain the IGMF based upon the $\mathrm{GeV}$ emission from blazars proceeds as follows: The beamed TeV blazar emission pair-creates off of the EBL. The resulting pairs subsequently up-scatter CMB photons to $\mathrm{GeV}$ energies. In principle, this should produce an observable $\mathrm{GeV}$ excess, or bump, in the spectra of these objects. However, in the presence of a large-scale IGMF, the ultra-relativistic pairs can be deflected significantly, directing the beamed $\mathrm{GeV}$ emission away from Earth. Thus, it is argued, the lack of a discernible $\mathrm{GeV}$ bump in a number of TeV blazars implies a lower limit upon the IGM field strength (as a function of $\mathrm{TeV}$ jet opening angle and variability timescale). The crucial components of the argument are

1. The TeV emission is beamed with the typical opening angles inferred from radio observations of AGN jets.

2. The variability timescale within the $\mathrm{TeV}$ source is long in comparison to the geometric time delays between the original $\mathrm{TeV}$ and inverse-Compton produced $\mathrm{GeV}$ gamma rays due to the orbit of the pairs through some angle $\vartheta, \Delta t \sim 10^{6}\left(D_{\mathrm{pp}} / 80 \mathrm{Mpc}\right)(\vartheta / 0.1 \mathrm{rad})^{2} \mathrm{yr}$ (see Dermer et al. 2011).

3. The pairs produced by $\mathrm{TeV}$ absorption on the EBL cool primarily via inverse-Compton scattering the CMB (and therefore evolve only due to inverse-Compton cooling and orbiting within the large-scale IGMF).

The first of these is supported indirectly by the lack of TeV emission from non-blazars. The implied TeV source stability required by the second is at odds with the variability observed in blazars at longer wavelengths. However, since the TeV-GeV delay is a strong function of deflection angle, given an empirical limit upon the $\mathrm{TeV}$ blazar variability timescale (presently $4 \mathrm{yr}$, Dermer et al. 2011) it is possible to produce a substantially weaker constraint upon the IGMF $\left(\sim 10^{-19} \mathrm{G}\right)$ (Dermer et al. 2011; Taylor et al. 2011; Takahashi et al. 2012).

Plasma cooling provides a fundamental limitation for these methods, however, by violating the third condition explicitly. Figures 3 and 4 imply that for all but the dimmest and highest-redshift $(z \gtrsim 4)$ gamma-ray blazars only a small fraction of the pair energy is lost to inverse-Compton on the CMB $\left(f_{\mathrm{IC}} \equiv \Gamma_{\mathrm{IC}} /\left(\Gamma_{\mathrm{IC}}+\Gamma_{\mathrm{M}, \mathrm{k}}\right)\right){ }^{13}$ In particular, the black lines in Figure 3 shows where $f_{\mathrm{IC}}=0.5$, i.e., roughly $50 \%$ of the TeVphoton power is ultimately converted into heat via plasma instabilities. As a result, the putative $\mathrm{GeV}$ component is typically much less luminous than otherwise expected, reducing the significance of non-detections substantially.

This may be seen explicitly in Figure 4, which shows $f_{\mathrm{IC}}$ as a function of gamma-ray flux for $E=1.85 \mathrm{TeV}$ (corresponding to a Comptonized-CMB photon energy of approximately $3 \mathrm{GeV}$ ), at number of source redshifts. Typical values for the

\footnotetext{
13 Here we implicitly assume that the nonlinear saturation of the plasma instabilities extract energy from the pair beam at the linear growth rate.
}

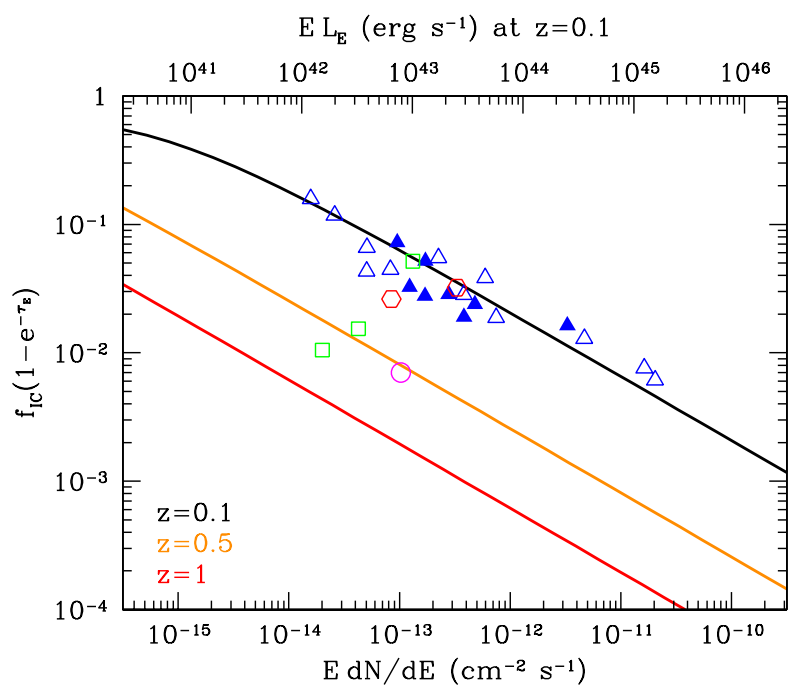

Figure 4. Fraction of the energy in the pair beam lost via inverseComptonization of the $\mathrm{CMB}$ at a number of different redshifts. In all cases the injection photon energy (as would have been observed at $z=0$ ) is $E=$ $1.85 \mathrm{TeV}$, resulting in $3 \mathrm{GeV}$ up-scattered $\mathrm{CMB}$ photons, the energy at which the Fermi/LAT instrument is most sensitive. For reference, the sources listed in Table 1 are also plotted (at $E=1 \mathrm{TeV}$ ), with $\mathrm{HBL}$, IBL, radio galaxies, and quasars shown by the the blue triangles, green squares, red hexagons and magenta circles, respectively. Filled points indicate sources that have been used to estimate the IGMF, corresponding to (in increasing flux) PKS 0548-322, 1ES 0347-121, 1ES 1101-232, RGB J0152+017, 1ES 0229+200, 1ES 1218+304, RGB J0710+591, and Mkn 501. For reference, the inferred isotropic luminosity is shown in the top axis for sources located at $z=0.1$.

TeV blazars collected in Table 1 (including those employed by Neronov \& Vovk 2010; Tavecchio et al. 2010, 2011; Dermer et al. 2011; Taylor et al. 2011; Takahashi et al. 2012; Dolag et al. 2011) lie in the range $10^{-3}$ to $3 \times 10^{-2}$, implying correspondingly small $\mathrm{GeV}$ Comptonization signals.

More importantly, when the beam evolution is dominated by plasma instabilities, over the inverse-Compton cooling timescale the pair distribution necessarily becomes isotropized. As a consequence, the angular distribution of the resulting $\mathrm{GeV}$ gamma rays (i.e., the orientation of the $\mathrm{GeV}$ "beam") is no longer indicative of the beam propagation through a large-scale magnetic field. That is, one expects large-angle deviations regardless of the IGMF strength.

Unfortunately, subject to the caveats of the preceding section, plasma instabilities appear to be the dominant cooling mechanism for the pair beams associated with the TeV blazars that have been used to constrain the IGMF thus far. The isotropic-equivalent luminosities of these sources range from $2.5 \times 10^{43} \mathrm{erg} \mathrm{s}^{-1}$ to $10^{45} \mathrm{erg} \mathrm{s}^{-1}$, placing them well within the plasma-instability dominated regime. As a result, the reported IGMF limits inferred from TeV blazars are presently unreliable.

Nevertheless, it may be possible to avoid the limitations imposed by plasma instabilities. At sufficiently low pair densities the cooling rates associated with the beam instabilities described in Section 3 fall below that due to inverse Compton. Moreover, at some point the plasma prescription breaks down altogether, suggesting that any plasma instabilities that operate on the skin-depth of the IGM are strongly suppressed. There are two distinct ways in which low pair densities can arise: low intrinsic luminosities and very short timescale events.

Below isotropic-equivalent luminosities of roughly $\sim$ 
$10^{42} \mathrm{erg} \mathrm{s}^{-1}$ inverse-Compton cooling dominates the beam instabilities we describe in Section 3 at $\sim 1 \mathrm{TeV}$. Below $10^{39} \mathrm{erg} \mathrm{s}^{-1}$ the plasma prescription itself breaks down altogether. While no TeV blazars with isotropic-luminosities below $10^{39} \mathrm{erg} \mathrm{s}^{-1}$ are known, there are a handful of very nearby sources below $10^{42} \mathrm{erg} \mathrm{s}^{-1}$. The two dimmest sources, the radio galaxies M87 and Cen A, however, are observable only due to their proximity (i.e., they have proper distances $\ll D_{\mathrm{pp}}$ ), intrinsically preventing them from providing a significant constraint on the IMGF. The highest source in Figure 4, and thus presumably the source with the largest fractional inverse-Compton signal, is PKS 2005-489, a dim, soft TeV blazar at $z=0.071$. Even in this case, however, the inverse-Compton cooling timescale is roughly 4 times longer than the plasma cooling timescale. Hence, probing the IGMF with TeV blazars will require observing considerably dimmer objects than those used thus far. Since doing so will likely require substantial increases in detector sensitivities ${ }^{14}$ we will not consider this possibility any further here.

Low pair densities may also be produced by limiting the duration of the VHEGR emission. Equation (5) was derived assuming that the pairs had reached a steady state between their formation via VHEGRs annihilating upon the EBL and their cooling via inverse-Compton and plasma processes. However, it takes roughly a cooling timescale for the pair beam densities to saturate at this level, which can be as long as $10^{5} \mathrm{yr}$ at some $E$ and $z$. Therefore, generally there is a lag between the onset of the VHEGR emission and the time at which plasma processes begin to dominate the cooling of the beam.

We can estimate this by setting $n_{b} \simeq 2 F_{E} \Delta t / D_{\mathrm{pp}}$ where $\Delta t$ is the duration of the emission. This is the case when the pair density is initially zero (i.e., it has been many cooling timescales since the last period of VHEGR emission) and $\Gamma \Delta t \ll 1$. Inserting this into the various cooling rates and setting $\Gamma_{\mathrm{M}, \mathrm{k}}=\Gamma_{\mathrm{IC}}$ gives an estimate for the maximum duration for which the pair density remains sufficiently low that inverse-Compton cooling dominates the cooling:

$$
\begin{aligned}
\Delta t \lesssim 5.3(1+\delta)^{1 / 2} & \left(\frac{1+z}{2}\right)^{(11-6 \zeta) / 2} \\
& \times\left(\frac{E L_{E}}{10^{45} \mathrm{erg} \mathrm{s}^{-1}}\right)^{-1}\left(\frac{E}{\mathrm{TeV}}\right)^{-2} \mathrm{yr},
\end{aligned}
$$

which may be found for the observed TeV sources in Table 1. Typically $\Delta t \simeq 300 \mathrm{yr}$ for the blazars that have been used to constrain the IGM, though $\Delta t$ ranges from $10^{2} \mathrm{yr}$ to $10^{4} \mathrm{yr}$ for TeV blazars generally. Note that it is not sufficient for emission to vary on $\Delta t$; such variations will be temporally smoothed on the much larger cooling timescale. Rather, it is necessary for the source to have been quiescent (i.e. isotropic-equivalent luminosity considerably less than $10^{42} \mathrm{erg} \mathrm{s}^{-1}$ ) for periods long in comparison to $\Gamma^{-1}$ and have turned on less than a time $\Delta t \ll$ $\Gamma^{-1}$ ago. As a consequence, only IGMF estimates using new $\mathrm{TeV}$ blazars can potentially avoid plasma instabilities in this way.

A natural example of a class of transient gamma-ray sources is gamma-ray bursts (GRBs). For GRBs an isotropic-

\footnotetext{
${ }^{14}$ As described in Section 5.1.2, the incompleteness of the TeV observations are well-described by a single flux limit, corresponding to an isotropicequivalent luminosity limit at $10^{2} \mathrm{Mpc}$ of roughly $10^{43} \mathrm{erg} \mathrm{s}^{-1}$. The flux limit of Fermi is estimated to be roughly $4 \times 10^{43} \mathrm{erg} \mathrm{s}^{-1}$ at $z \simeq 0.1$ (see Figure 23 of Abdo et al. 2010c).
}

equivalent energy of roughly $10^{54} \mathrm{erg}$ is required for the plasma instabilities to grow efficiently, comparable to the total energetic output of the brightest events. Using GRBs to probe the IGMF has been suggested previously, and limits based upon the lack of a delayed GeV component in some GRBs already exist, finding field strengths above $\sim 10^{-22} \mathrm{G}$ (see, e.g., Plaga 1995; Dai \& Lu 2002; Guetta \& Granot 2003; Takahashi et al. 2008). Future efforts should be able to probe field strengths of $10^{-15} \mathrm{G}$ for $z \lesssim 0.2$ (see Figure 4 of Takahashi et al. 2008). This is predicated, however, upon the existence of a significant VHEGR component in the prompt or afterglow emission of GRBs.

Ando \& Kusenko (2010) have attempted to directly detect the $\mathrm{GeV}$ signal in the vicinity of AGNs that do not exhibit $\mathrm{TeV}$ emission. That is, since an IGMF can in principle induce large-angle deviations in the propagation direction of the upscattered $\mathrm{GeV}$ photon relative to the original VHEGR, one may search for a $\mathrm{GeV}$ excess at large distances from non-blazars. This is made much more difficult by the extraordinarily low surface gamma-ray brightness due to the large $D_{\mathrm{pp}}$, random source orientations, and potential dilution associated with the beam diffusion. Nevertheless, Ando \& Kusenko (2010) claim a marginal detection of this component in stacked Fermi images, though the Fermi point-spread function appears to be capable of completely explaining their result (Neronov et al. 2011). If such a $\mathrm{GeV}$-halo were found around $\mathrm{TeV}$-bright sources with the anticipated luminosity, it would argue strongly against plasma instabilities dominating the beam evolution. However, we note that for many TeV-dim sources inverse-Compton cooling still dominates the beam evolution, and thus in stacked images of these sources GeV-halos may still be present.

Note that, as discussed in Section 3.6, a strong IGMF $(\sim$ $10^{-12} \mathrm{G}$ ) could, in principle, suppress the growth of the plasma beam instabilities significantly. As we argue there, a sufficiently pervasive and strong IGMF would necessarily be primordial in origin. However, few constraints upon such a primordial field exist, especially since we have argued that the lack of an inverse Compton $\mathrm{GeV}$ bump cannot be used as a constraint on the IGMF. Hence, we are left with constraints from Big Bang Nucleosynthesis $\left(B \lesssim 10^{-7} \mathrm{G}\right.$, Kandus et al. 2011, and references therein), from the CMB $\left(B \lesssim 10^{-9} \mathrm{G}\right.$, Barrow et al. 1997), and limits on the Faraday rotation of distance radio sources $\left(B \lesssim 10^{-9} \mathrm{G}\right.$, Vallée 2011 , and references therein). A stronger limit can be obtained from limits upon the deflection angle $\left(<2^{\circ}\right)$ of ultra-high energy cosmic rays $\left(E \gtrsim 10^{20} \mathrm{eV}\right)$ from distant sources at a distance $d>\lambda_{B}$, which upon assuming a magnetic correlation length, $\lambda_{B}$, gives $B \lesssim 10^{-11}\left(E / 10^{20} \mathrm{eV}\right)\left(\lambda_{B} / 100 \mathrm{Mpc}\right)^{-1}\left(d / \lambda_{B}\right)^{-1 / 2} \mathrm{G}$ (see for instance Waxman \& Miralda-Escude 1996; de Angelis et al. 2008), though this depends strongly upon the assumed structure of the IGMF and small-scale fields may be significantly stronger than this (Kotera \& Lemoine 2008). Proposed mechanisms by which a primordial IGMF can be theoretically generated are essentially unconstrained, yielding B-field strengths between $B \sim 10^{-9}-10^{-30} \mathrm{G}$ (Kandus et al. 2011).

However, in the remainder of this work and in Paper II and Paper III, we show that the effect of the "oblique" (or similar) instability may potentially explain many different observed phenomena in cosmology and high energy astrophysics. Taking the beam instabilities we present here at face value, that so many observational puzzles can be simultaneously explained by the local dissipation of $\mathrm{TeV}$ blazar emission would im- 
ply the strongest upper limit upon primordial magnetism to date. Namely, the apparent impact of TeV blazars upon the large-scale cosmological environment places a constraint on the IGMF of $B \lesssim 10^{-12} \mathrm{G}$.

\section{IMPLICATIONS FOR THE BLAZAR LUMINOSITY FUNCTION}

The EGRB corresponds to the diffuse gamma-ray background $(E>10 \mathrm{MeV})$ not associated with Galactic sources. This has been measured at high energies by EGRET (Sreekumar et al. 1998; Strong et al. 2004) and more recently strongly constrained between $200 \mathrm{MeV}$ and $100 \mathrm{GeV}$ by Fermi (Abdo et al. 2010b,a). The most recent Fermi estimate of the EGRB is a featureless power-law, somewhat softer than that found by EGRET, and does not exhibit the localized high-energy excess claimed by a reanalysis of the EGRET data (Strong et al. 2004).

While bright, nearby blazars are resolved, and therefore excluded from the EGRB, the remainder is thought to arise nearly exclusively from distant gamma-ray emitting blazars, upon which the Fermi EGRB places severe constraints (Abdo et al. 2010b). Beyond $z \simeq 0.25$ Fermi cannot detect blazars with isotropic-equivalent luminosities comparable to the objects listed in Table 1, and thus the vast majority of such objects contribute to the Fermi EGRB.

A significant EGRB above $100 \mathrm{GeV}$ is not expected due to annihilation upon the EBL. However, if they operate efficiently, ICCs can reprocesses the VHEGR emission of distant sources into the Fermi EGRB energy range (i.e., $\lesssim 100 \mathrm{GeV}$ ). Thus, a number of efforts to constrain the VHEGR emission from extragalactic sources based upon the EGRB can be found in the literature (Narumoto \& Totani 2006; Kneiske \& Mannheim 2008; Inoue \& Totani 2009). These have typically found that the comoving number density of VHEGR-emitting blazars could not have been much higher at high- $z$ than it is today. Even with moderate evolutions (e.g., that in Narumoto \& Totani 2006; Inoue \& Totani 2009) consistent with the EGRET EGRB, substantially over-produce the Fermi EGRB, and are therefore believed to be excluded (Venters 2010).

However, here we show that this conclusion is predicated upon the high-efficiency of the ICC. We have already shown that for bright VHEGR sources plasma beam instabilities extract the kinetic energy of the first generation of pairs much more rapidly than inverse-Compton scattering. As a consequence, the ICC is typically quenched, substantially limiting the contributions of these sources to the EGRB. Thus, even with a dramatically evolving blazar population, e.g., similar to that of quasars, it is possible for these objects to be consistent with the Fermi EGRB. While our discussion of the EGRB and the evolution of blazars is predicated on the extraction of the pair-beam kinetic energy by plasma beam instabilities, our conclusions are not. Rather they will continue to hold as long as the pair beams are locally dissipated - via plasma beam instabilities or some other equally powerful mechanism.

\subsection{An Empirical Estimate of the TeV Blazar Luminosity Function}

Blazars dominate the extragalactic gamma-ray sky, and thus represent the best studied VHEGR source class. Since we are interested exclusively in those objects responsible for the bulk of the VHEGR emission, we necessarily concentrate upon the subset of blazars that are luminous VHEGR emitters. Of the $28 \mathrm{TeV}$ sources listed in Table 1, 23 are peaked at very-high energies (the HBL and hard IBL sources, for a full definition see below), and comprise what we will call collectively $\mathrm{TeV}$ blazars. This necessarily is limited to $z \simeq 0.1$ as a consequence of the annihilation of VHEGRs upon the EBL. Nevertheless, we will find that this is remarkably similar to the local quasar luminosity function $\left(\phi_{Q}\right)$, and thus attempt to construct a blazar luminosity function by analogy:

$$
\phi_{B}(z, L) \equiv \frac{d^{2} \Phi_{B}}{d z d \log _{10} L},
$$

in which $\Phi_{B}$ is the comoving number density of blazars at redshifts less than $z$ with isotropic-equivalent luminosities below $L$. This is different from previous efforts to empirically constrain $\phi_{B}$ from the EGRB (see, e.g., Narumoto \& Totani 2006; Inoue \& Totani 2009) in at least two ways. First, we begin with an empirically determined local $\phi_{B}$ and attempt to extend this by placing the $\mathrm{TeV}$ blazars within the broader context of accreting supermassive black holes, rather than beginning with the EGRB and working backwards to infer an acceptable $\phi_{B}$. Second, we are primarily concerned with $\phi_{B}$ of TeV blazars, and specifically do not consider the contributions from other kinds of objects. While this does not represent a significant oversight in terms of the high-energy contributions to the EGRB, which almost certainly arises from the VHEGR-emitting blazars, it does mean that our conclusions regarding the luminosity function of the TeV blazars do not necessarily apply to all Fermi AGN (e.g., the FSRQs, see below).

\subsubsection{Placing TeV blazars in Context}

The TeV blazars presumably fit within the broader context of the blazars observed by Fermi specifically, and AGNs generally. For this reason, here we briefly review the physical classification scheme based on the widely accepted AGN standard paradigm that provides a unified picture of the emission emission properties of these objects (e.g., Urry \& Padovani 1995). Specifically, we summarize the classes of objects believed to be capable of producing significant $\mathrm{TeV}$ luminosities and the potential physical processes responsible for the observed emission. Based upon these we then assess the implications for the number, variability, and redshift evolution of the TeV blazars.

In general there exist two main classes of AGNs that differ in their accretion mode and in the physical processes that dominate the emission.

1. Thermal/disk-dominated AGNs: Infalling matter assembles in a thin disk and radiates thermal emission with a range of temperatures. The distributed black-body emission is then Comptonized by a hot corona above the disk that produces power-law X-ray emission. Hence the emission is a measure of the accretion power of the central object. This class of objects are called QSOs or Seyfert galaxies and make up about $90 \%$ of AGNs. They preferentially emit in the optical or X-rays and do not show significant nuclear radio emission. None of these sources have so far been unambiguously detected by Fermi or imaging atmospheric Cherenkov telescopes because the Comptonizing electron population is not highly relativistic and emits isotropically, i.e. there is no beaming effect that boosts the emission.

2. Non-thermal/jet-dominated AGNs: The non-thermal emission from the radio to X-ray is synchrotron emission in a magnetic field by highly energetic electrons that have been accelerated in a jet of material ejected from the nucleus at relativistic speed. The same population of electrons can also Compton up-scatter any seed photon 
population either provided by the synchrotron emission itself or from some other external radiation field such as UV radiation from the accretion disk. Hence the SED of these objects shows two distinct peaks. The luminosity of these non-thermal emission components probes the jet power of these objects. Observationally, this leads to the class of radio-loud AGNs which can furthermore be subdivided into blazars and non-aligned non-thermal dominated AGNs depending on the orientation of their jets with respect to the line of sight.

There are no known sources above $600 \mathrm{GeV}$ that correspond to AGNs with jets pointed at large angles $\left(\approx 15^{\circ}-40^{\circ}\right.$, see Urry \& Padovani 1995) with respect to the line of sight (for an example of a non-aligned AGN, NGC1275, that shows a very steep high-energy spectrum, emitting a negligible number of VHEGRs, see Mariotti \& MAGIC Collaboration 2010). Hence we turn our attention to blazars, which can be powerful TeV sources.

Blazars can further be subdivided into two main subclasses depending upon their optical spectral properties: flat spectrum radio quasars (FSRQ) and BL Lacs. FSRQs, defined by broad optical emission lines, have SEDs that peak at energies below $1 \mathrm{eV}$, implying a maximum particle energy within the jet and limiting the inverse-Compton scattered photons mostly to the soft gamma-ray band. It is presumably for this reason that no continuous TeV component has been detected in an FSRQ (note, however, that TeV flares from FSRQs have been detected in two cases (MAGIC Collaboration et al. 2008b; Mose Mariotti 2010)).

In contrast, BL Lacs or Blazars of the BL Lac type (Massaro et al. 2009) can be copious TeV emitters. These are very compact radio sources and have a broadband SED similar to that of strong lined blazars, though lack the broad emission lines that define those. Depending upon the peak energy in the synchrotron spectrum, which approximately reflects the maximum particle energy within the jet, they are classified as low-, intermediate-, or high-energy peaked BL Lacs, respectively called LBL, IBL, and HBL (Padovani \& Giommi 1995; Abdo et al. 2010d). ${ }^{15}$ While LBLs peak in the far-IR or IR band, they exhibit a flat or inverted X-ray spectrum due to the dominance of the inverse-Compton component (see Fig 15 of Abdo et al. 2010d, for a visualization of the SED of BL Lacs). The synchrotron component of IBLs peaks in the optical which moves their inverse-Compton peak into the gamma-ray band of Fermi. HBLs are much more powerful particle accelerators, with the synchrotron peak reaching into the UV or, in some cases, the soft X-ray bands. The inverse-Compton peak can then reach TeV energies (Ghisellini \& Tavecchio 2008; Tavecchio \& Ghisellini 2008; Abdo et al. 2010d). ${ }^{16}$

In the gamma-ray band, the subclass of IBLs that emit VHEGRs are almost indistinguishable from the HBLs, suggesting that the location of the synchrotron peak does not uniquely characterize the VHEGR emission from these sources (e.g., due to variations among individual blazars in the magnetic field strength within the synchrotron emitting region and the origins

\footnotetext{
15 The source classes of HSP/ISP/LSP used in recent Fermi publications are very similar to the commonly used HBL/IBL/LBL classes. Hence we identify these with each other, respectively, though minor differences may be found in the literature. Nevertheless, where we refer to the number counts observed by Fermi specifically, we will refer to the classes HSP/ISP/LSP in keeping with their notation.

${ }^{16}$ For the highest energies scattering occurs in the Klein-Nishina regime, which results in steeper inverse-Compton spectra.
}

and properties of the seed photons that are ultimately Comptonized). Hence we identify HBLs and VHEGR-emitting IBLs with the single source class of TeV blazars. We note that there is presently no evidence for the hypothetical class of ultraHBLs that were proposed to have a very energetic synchrotron component extending to $\gamma$-rays (Ghisellini 1999). If such a population of bright and numerous sources exists, Fermi should have seen it (Abdo et al. 2010c). The ultra-HBLs may have escaped detection from Fermi thus far by being either intrinsically $\operatorname{dim} \gamma$-ray sources or very rare objects (Costamante et al. 2007).

TeV blazars have a redshift distribution that is peaked at low redshifts extending only up to $z=0.7$. This is most likely entirely a flux selection effect; $\mathrm{TeV}$ blazars are intrinsically less luminous than LBLs and FSRQs, with an observed isotropicequivalent luminosity range of $10^{44}-2 \times 10^{46} \mathrm{erg} \mathrm{s}^{-1}$, with the highest redshift $\mathrm{TeV}$ blazars also being among the most luminous objects (see Figures 23 and 24 in Abdo et al. 2010c). That TeV blazars should be intrinsically less luminous than FSRQs is not entirely unexpected, however. Ghisellini et al. (2009) have argued that the physical distinction between FSRQs and $\mathrm{TeV}$ blazars has its origin in the the different accretion regimes of the two classes of objects. Using the gamma-ray luminosity as a proxy for the bolometric luminosity, the boundary between the two subclasses of blazars can be associated with the accretion rate threshold (nearly $1 \%$ of the Eddington rate) separating optically thick accretion disks with nearly Eddington accretion rates from radiatively inefficient accretion flows. The spectral separation in hard (BL Lacs) and soft (FSRQs) objects then results from the different radiative cooling suffered by the relativistic electrons in jets propagating into different surrounding media (Ghisellini et al. 2009). Hence in this model, TeV blazars cannot reach higher luminosities than approximately $2 \times 10^{46} \mathrm{erg} \mathrm{s}^{-1}$ since they are limited by the nature of inefficient accretion flows that power these jets and by the maximum black hole mass, $\sim 10^{10} M_{\odot}$.

\subsubsection{An Empirical Local $\phi_{B}(z, L)$}

While we have attempted to place the observed TeV blazars, which we have identified with the HBLs and VHEGR-emitting IBLs, into the broader context of AGNs using the unified model, due to the substantial distinctions in accretion rate, emission properties, object morphology and geometry, it is not obvious that any of the properties of TeV blazars should be similar to those of AGNs more generally. Nevertheless, evidence for a simple connection between the two populations can be found in the similarity between their the luminosity functions (a fact we will exploit later in estimating the redshift evolution of the TeV blazars). Here we define the luminosity for the purposes of defining $\phi_{B}$ to be the isotropic-equivalent value associated with emission between $100 \mathrm{GeV}$ and $10 \mathrm{TeV}$. While this may be considered to be a VHEGR luminosity, because most TeV blazars are peaked within this band, this corresponds to the majority of the emission from these sources.

The objects listed in Table 1 were chosen because they have well defined SEDs, based upon a combination of VERITAS, H.E.S.S., and MAGIC observations. These 28 sources have VHEGR spectra that are well fit by the form,

$$
\frac{d N}{d E}=f_{0}\left(\frac{E}{E_{0}}\right)^{-\alpha},
$$

where $f_{0}$ is the normalization in units of $\mathrm{cm}^{-2} \mathrm{~s}^{-1} \mathrm{TeV}^{-1}$. The gamma-ray energy flux is trivially related to $d N / d E$ by $F_{E}=$ 


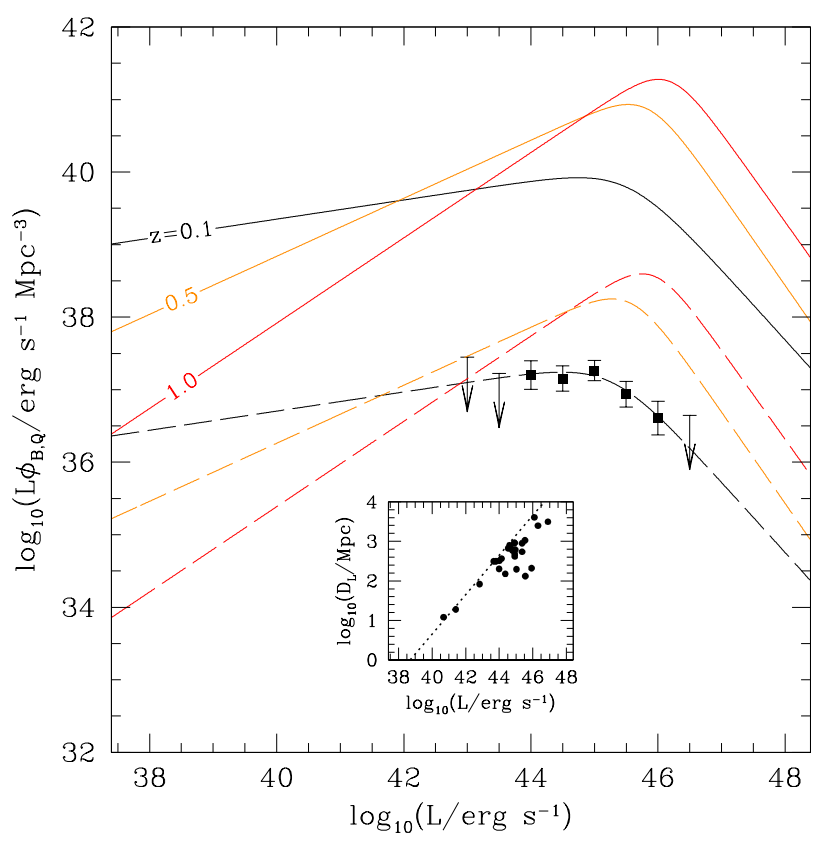

Figure 5. Comparison between the luminosity-weighted quasar and TeVblazar luminosity functions $\left(L \phi_{Q}(z, L)\right.$ and $L \phi_{B}(z, L)$, respectively). The solid lines show the absolute $L \phi_{Q}$ (in comoving Mpc), while the dashed lines show $L \phi_{Q}$ rescaled in magnitude by $2.1 \times 10^{-3}$ and shifted to lower luminosities by a factor of 0.55 . In both cases the black, orange, and red lines correspond to $\phi_{Q}(0.1, L), \phi_{Q}(0.5, L)$, and $\phi_{Q}(1, L)$, respectively. The points and upper-limits show $\phi_{B}$ of the HBL and IBL sources listed in Table 1, assuming the relevant limits for PKS $1553+113$ and PKS $1424+240$. Presented in the inset is the TeV source luminosity distance as a function of source luminosity for all of the sources in Table 1 with redshift estimates (including limits). The dotted line shows the distance-dependence of the flux limit we employ in the completeness correction.

$E d N / d E \propto E^{1-\alpha}$, from which we obtain a VHEGR flux,

$$
F=E_{0} f_{0} \int_{100 \mathrm{GeV}}^{10 \mathrm{TeV}} d E\left(\frac{E}{E_{0}}\right)^{1-\alpha}
$$

and for sources with a measured redshift a corresponding isotropic-equivalent luminosity, $L=4 \pi D_{L}^{2} F$, where $D_{L}$ is the luminosity distance. ${ }^{17}$ The resulting $f_{0}, E_{0}, \alpha, F$, and $L$ are collected in Table 1. In addition we list the redshift, inferred comoving distance, and absorption-corrected intrinsic spectral index, defined at $E_{0}$, obtained via

$$
\hat{\alpha}=-\left.\frac{d \ln E^{-\alpha} e^{\tau_{E}[E(1+z), z]}}{d \ln E}\right|_{E_{0}} \simeq \alpha-\tau_{E}\left[E_{0}(1+z), z\right] .
$$

For high-redshift sources $\hat{\alpha}$ can be substantially less than 2 , implying that an intrinsic spectral upper-cutoff must exist.

To produce $\phi_{B}$, we must account for a variety of selection effects inherent in the sample listed in Table 1. The objects in Table 1 were originally selected for study for a variety of sourcespecific reasons, e.g., existing well known sources, extremely high X-ray to radio flux ratio in the Sedentary High energy peaked BL Lac catalog, hard spectrum sources in the Fermi point source catalog, and flagged as promising by the FermiLAT collaboration. In addition, the source selection suffered from the usual problems associated with surveys (e.g., scheduling conflicts with other targets, moon, bad weather, etc.). As a

\footnotetext{
17 Since the VHEGR spectra of TeV blazars typically are peaked above $100 \mathrm{GeV}$, this overestimates the luminosity by a factor of order unity.
}

consequence, this sample is somewhat inhomogeneous. Nevertheless, in lieu of a less-biased sample, we will treat it as homogeneous and correct for the selection effects were possible, focusing upon those due to the sky coverage and duty cycle of $\mathrm{TeV}$ observations, and those due to sensitivity limits of current imaging atmospheric Cerenkov telescopes.

To estimate the sky completeness and duty cycle of this set of objects we rely upon the all-sky GeV gamma-ray observations of HBL and IBL sources by Fermi (Abdo et al. 2010c). Outside of the Galactic plane, Fermi observes 118 high-synchrotron peaked (HSP) blazars and a total of 46 intermediate-synchrotron peaked (ISP) blazars. Roughly half of the latter are likely to emit VHEGRs as indicated by their flat spectral index between 0.1 and $100 \mathrm{GeV}(\alpha \lesssim 2$; see the spectral index distribution of Figure 14 in Abdo et al. (2009)). Of these potential $141 \mathrm{TeV}$ blazars, only 22 have also been coincidentally identified as $\mathrm{TeV}$ sources, whereas there are a total of 33 known TeV blazars (29 HBL, 4 IBL). If these 141 sources are all $\mathrm{TeV}$ emitters, but have not been detected due to incomplete sky coverage of current $\mathrm{TeV}$ instruments, then the selection factor is $\eta_{\text {sel }}=141 / 33=4.3$. In addition, the duty cycle of coincident $\mathrm{GeV}$ and $\mathrm{TeV}$ emission is $\eta_{\text {duty }}=33 / 22=1.5$. $^{18}$ Finally, by excluding the Galactic plane for galactic latitudes $b<10^{\circ}$, this is an underestimate by roughly $\eta_{\text {sky }}=1.17$.

We make a rough attempt to correct for the sensitivity limit of the $\mathrm{TeV}$ instruments, inferring a flux limit by fitting the upperenvelope of the TeV-blazar flux-luminosity distance distribution. Somewhat surprisingly, despite the heterogeneous nature of the $\mathrm{TeV}$ observations, are remarkably well described by a single flux limit: $4.19 \times 10^{-12} \mathrm{erg} \mathrm{cm}^{-2} \mathrm{~s}^{-1}$. This process and the associated limit are shown explicitly in the inset of Figure 5. From this we obtain a maximum redshift, and therefore comoving volume, associated with each luminosity. We then construct $\phi_{B}$ by counting the number of TeV blazars per unit $\log _{10} L$ in each logarithmic luminosity bin and dividing by the comoving volume. The resulting $\phi_{B}$, weighted by luminosity (and therefore showing the luminosity density in comoving units), is shown in Figure 5. It peaks at $\sim 4 \times 10^{44} \mathrm{erg} \mathrm{s}^{-1}$, implying that, as expected, these objects are systematically dimmer than most other AGN, and exhibits the broken-power law shape typical of AGN luminosity functions. More importantly, despite a handful of sources with redshifts $\sim 0.5$, the objects in Table 1 are all nearby, and therefore our $\phi_{B}$ corresponds to that in the local Universe, i.e., $z \sim 0.1$. Based upon this, the inferred present-day TeV-blazar luminosity density is roughly $1 \times 10^{38} \mathrm{erg} \mathrm{s}^{-1} \mathrm{Mpc}^{-3}$.

Also shown in Figure 5 is the $L \phi_{Q}$ obtained by Hopkins et al. (2007). After rescaling $L \phi_{Q}$ to lower luminosities (0.55) and lower luminosity densities $\left(2.1 \times 10^{-3}\right)$, it provides a remarkably good fit to our $L \phi_{B}$ (reduced- $\chi^{2}$ of 0.13 with 3 degrees of freedom), i.e., we find

$$
\phi_{B}(0.1, L) \simeq 3.8 \times 10^{-3} \phi_{Q}(0.1,1.8 L),
$$

where an explicit expression for $\phi_{Q}$ is given in Appendix B. While there is considerable uncertainty in $L \phi_{B}$, especially at low luminosities, it clearly does not fit $L \phi_{Q}$ at higher $z$. This suggests two immediate conclusions:

\footnotetext{
18 Note that we implicitly assume that the luminosity distribution of observed $\mathrm{TeV}$ sources reflects the true distribution after accounting for flux incompleteness, and thus constant correction factors (independent of luminosity) can be used to estimate the true distribution. We show that this assumption is fulfilled in Figure 5.
} 


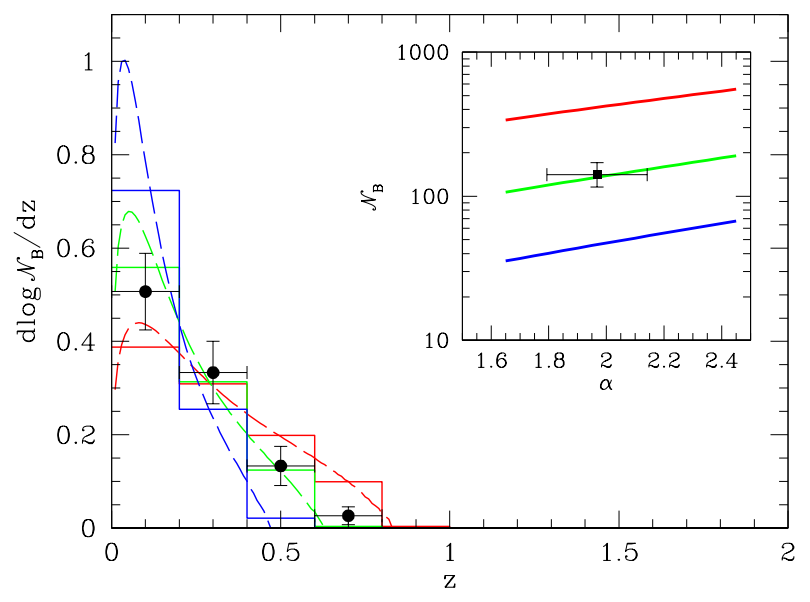

Figure 6. The normalized number of TeV blazars (presumably the hard gamma-ray blazars comprised of hard ISPs and all HSPs) that are expected to have been observed by Fermi as a function of redshift. The dashed lines and solid histograms show the ideal and binned distributions for $\alpha=1.95$, though neither varies significantly with $\alpha$. The total number of objects is sensitive to the spectral index, and shown as a function of $\alpha$ in the inset. Different colors correspond to different normalizations between the $100 \mathrm{GeV}-10 \mathrm{TeV}$ and $100 \mathrm{MeV}-100 \mathrm{GeV}$ luminosities: $\eta_{\min }=0.78$ (red), 1.6 (green), and 3.1 (blue) i.e., the former is the latter luminosity multiplied by $\eta_{\min }$ ). For reference, the normalized number of hard gamma-ray blazars (HSPs and half of the ISPs) observed by Fermi are shown by the black circles, and the black square in the inset shows the average spectral index and total number observed, with horizontal error bars giving the $1-\sigma$ range.

1. The bolometric output of TeV blazars and quasars are regulated by similar mechanisms, presumably accretion, despite the large difference in luminosity and the details of the emission processes between the two populations.

2. TeV blazars and quasars are contemporaneous elements in a single AGN distribution; specifically, TeV-blazar activity does not lag that of quasars.

\subsubsection{Extending $\phi_{B}(z, L)$ to high $z$}

Based upon the strong similarities between $\phi_{B}$ and $\phi_{Q}$, and the associated implications, we make the conservative theoretical assumption that the redshift evolution of the TeV blazars follows that of quasars. That is, we suppose that Equation (29) holds at all $z$. This implies that the integrated comoving TeVblazar isotropic-equivalent luminosity density is given by

$$
\Lambda_{B}(z) \equiv \int_{-\infty}^{\infty} L \phi_{B}(z, L) d \log _{10} L=\eta_{B} \Lambda_{Q}(z),
$$

where the constant of proportionality, $\eta_{B} \simeq 2.1 \times 10^{-3}$, is then set by the comparison between $\phi_{B}$ and $\phi_{Q}$ at $z=0.1$. As a consequence, in comoving units, the TeV-blazar luminosity density would be roughly an order of magnitude larger at $z \simeq 2$, $\sim 1 \times 10^{39} \mathrm{erg} \mathrm{s}^{-1} \mathrm{Mpc}^{-3}$.

\subsection{Fermi TeV Blazar Counts and the Local Evolution of the Blazar Luminosity Function}

The redshift distribution of the Fermi BL Lac sample (i.e., the First LAT Catalog, 1LAC; Abdo et al. 2010c) is peaked at $z<0.2$ and falls rapidly thereafter. Inasmuch as the Fermi HSP and ISP counts directly probes the low- $z \phi_{B}$, it may appear that they are inconsistent with the rapidly evolving $\phi_{B}$ described in the previous section. Indeed, an analysis of the first three months of Fermi observations, suggested that the BL Lac population does not grow substantially with redshift (Abdo et al. 2009). However, as seen in Figure 6, this is not necessarily the case.

Assuming, as we have, that the number of $\mathrm{TeV}$ blazars is equal to the the number of hard Fermi gamma-ray blazars, corresponding to ISPs with $\alpha \lesssim 2$ (comprising roughly half of that class) and HSPs, the expected number observed inside of a given redshift is:

$$
\begin{aligned}
\mathcal{N}_{B}(z)=\int_{0}^{z} d z^{\prime} & \frac{d D}{d z^{\prime}} 4 \pi D_{A}^{2} \\
& \times \int_{\log _{10} L_{\min }\left(z^{\prime}\right)}^{\log _{10} L_{\max }}\left(1+z^{\prime}\right)^{3} \phi_{B}\left(z^{\prime}, L\right) \mathrm{d} \log _{10} L
\end{aligned}
$$

where $D \equiv \int c d t^{\prime}=\int c d z^{\prime} /\left[H\left(z^{\prime}\right)\left(1+z^{\prime}\right)\right]$ and $D_{A}=D_{L} /(1+$ $z)^{2}$ are the proper and angular diameter distances, respectively, $L_{\max } \simeq 2 \times 10^{46} \mathrm{erg} \mathrm{s}^{-1}$ is the intrinsic upper-cutoff of the TeV blazar isotropic-equivalent luminosity function, and

$$
\begin{aligned}
L_{\min }(z) & =F_{\min } 4 \pi D_{L}^{2}(z)(1+z)^{\alpha-2} \\
& \simeq 4 \times 10^{46} \eta_{\min }\left(\frac{1+z}{2}\right)^{\alpha-2}\left[\frac{D_{L}(z)}{D_{L}(1)}\right]^{2} \operatorname{erg~s}^{-1}
\end{aligned}
$$

is lower-limit set by the Fermi flux limit (see Figure 23 of Abdo et al. 2010c, and surrounding discussion). The factor $\eta_{\min }$ is a correction relating the $100 \mathrm{GeV}-10 \mathrm{TeV}$ isotropic-equivalent luminosities we employ to define $\phi_{B}$ to the $100 \mathrm{MeV}-100 \mathrm{GeV}$ luminosities used by Fermi to define the flux limit. The dependence upon $\alpha$ arises from the limited spectral coverage of both definitions, though here we fix $\alpha=3$ for all objects based upon the sources that dominate the $\mathrm{TeV}$ flux at Earth. Note that since the $\phi_{Q}$ from Hopkins et al. (2007) diverges at small $L$, the lower-luminosity cutoff is critical to getting both the total number of TeV blazars and the shape of their redshift distribution correct. The resulting $\mathcal{N}_{B}$ is shown in Figure 6 for three different choices of $\eta_{\min }: 0.78,1.6$, and 3.1, of which $\eta_{\min }=1.6$ is most similar to the $1 \mathrm{LAC}$ hard gamma-ray blazars.

Generally, our $\phi_{B}$ does an excellent job of reproducing the overall number of 1LAC hard gamma-ray blazars and the dominance of nearby objects in their redshift distribution. This is despite the strong redshift evolution of $\phi_{B}$ implied by its relationship with $\phi_{Q}$. The reason for this is the flat distribution at low $L$, the steep drop-off at high $L$ (a result of which is that the shape is only marginally sensitive to the cutoff at $L_{\max }$ ) and the rapidly growing $L_{\min }$ due to the fixed flux limit.

However, we note that the comparison between $\phi_{B}$ and the 1LAC hard gamma-ray blazar statistics assumes that those with measured redshifts, comprising roughly half of the 1LAC hard gamma-ray blazar sample, are representative of the hard gamma-ray blazar population as a whole. This appears not to be the case; as we discuss in detail in Appendix $\mathrm{C}$, it is clear that the 1LAC HSPs with and without measured redshifts are not drawn from the same underlying $\alpha$-distribution. Based upon a similar analysis for 1LAC BL Lacs generally, Abdo et al. (2010c) argued that the objects without redshifts are more consistent with $z>0.5$ population. However, this conclusion does not extend to HSPs, for which there are only three sources in the clean 1LAC HSP sample with $z>0.5$. Rather, in Appendix C, we show that the 1LAC HSPs without redshifts are distributed both in spectral index and flux much 
more similarly with nearby HSPs $(z<0.25)$ than more distant HSPs $(z \geq 0.25)$. If this is because these sources are intrinsically under-luminous, nearby objects, it could marginally improve the already remarkable comparison between the number of objects implied by our $\phi_{B}$ and those observed. However, because the number of predicted 1LAC hard gamma-ray blazars is strongly dependent upon the flux limit, even a marginal increase in either $\phi_{B}$ at low luminosities or the effective flux limit results in a $d \mathcal{N}_{B} / d z$ that is more strongly weighted at low $z$, potentially allowing even more dramatically evolving luminosity functions. For this reason we conclude that our $\phi_{B}$ is broadly consistent with the 1LAC hard gamma-ray blazar distribution, and that Fermi does not, at present, exclude a rapidly evolving $\phi_{B}$ in the recent past.

\subsection{The TeV Blazar Contribution to the Fermi EGRB}

While the statistics of the 1LAC hard gamma-ray blazar sample probes the evolution of $\phi_{B}$ at $z \lesssim 0.5$, the Fermi EGRB is sensitive to $\mathrm{TeV}$ Blazars at high $z$. The contribution to the EGRB from TeV blazars given our $\phi_{B}$ is shown in Figure 7. Because ICCs are suppressed, computing the EGRB flux requires only summing the individual intrinsic $\mathrm{GeV}$ spectra of the $\mathrm{TeV}$ blazars. For simplicity, to estimate the TeV blazar contribution to the EGRB we assume all TeV blazars have identical intrinsic spectra, given by a broken power law,

$$
F_{E}=L \hat{F}_{E} \propto \frac{1}{\left(E / E_{b}\right)^{\alpha_{L}-1}+\left(E / E_{b}\right)^{\alpha-1}},
$$

where the normalization is set such that the $\int_{100 \mathrm{GeV}}^{10 \mathrm{TeV}} \hat{F}_{E} d E=1$ (i.e., $\int_{100 \mathrm{GeV}}^{10 \mathrm{TeV}} L \hat{F}_{E} d E=L$, corresponding to the luminosity used to define $\phi_{B}$, and thus determine $\left.\eta_{B}\right), E_{b}$ is the energy of the spectral break, and $\alpha_{L}<\alpha$ are the low and high-energy spectral indexes, respectively. ${ }^{19}$ With this, after performing the integral over $L$, the EGRB flux is then

$$
\begin{aligned}
E^{2} \frac{d N}{d E}(E, z) & =\frac{\eta_{B}}{4 \pi} \int_{z}^{\infty} d z^{\prime} \frac{d D}{d z^{\prime}} 4 \pi D_{A}^{2} \frac{\tilde{\Lambda}_{Q}\left(z^{\prime}\right) \hat{F}_{E^{\prime}}}{4 \pi D_{L}^{2}} \mathrm{e}^{-\tau_{E}\left[E^{\prime}, z^{\prime}\right]} \\
& =\frac{\eta_{B}}{4 \pi} \int_{z}^{\infty} d z^{\prime} \frac{c \tilde{\Lambda}_{Q}\left(z^{\prime}\right)}{H\left(z^{\prime}\right)\left(1+z^{\prime}\right)^{5}} \hat{F}_{E^{\prime}} \mathrm{e}^{-\tau_{E}\left[E^{\prime}, z^{\prime}\right]},
\end{aligned}
$$

where $E^{\prime}=E\left(1+z^{\prime}\right)$, and $\tilde{\Lambda}_{Q}=\Lambda_{Q}(1+z)^{3}$ is the physical quasar luminosity density. We choose fiducial values for the spectral parameters of $\alpha_{L}=1.33, E_{b}=1 \mathrm{TeV}$, and $\alpha=3$, typical of the TeV blazars (see Table 1 and Ghisellini 2011). Finally, since Fermi resolves individual gamma-ray blazars with isotropicequivalent luminosities $\sim 10^{45} \mathrm{erg} \mathrm{s}^{-1}$, roughly the location of the peak in $\phi_{B}$, for $z \lesssim 0.25$, we construct the EGRB from sources at larger redshift.

If $E_{b}$ is sufficiently high $\left(\gtrsim 2 \times 10^{2} \mathrm{GeV}\right)$ the EGRB is remarkably insensitive to the particular values of $E_{b}$ and $\alpha$ (see the bottom two panels of Figure 7). This is a direct result of the annihilation of the VHEGRs upon the EBL, effectively removing the relevant portion of the blazar SED from the EGRB. More important is the low-energy spectral index. However, for values of $\alpha_{L}$ that are consistent with the Comptonization models for the VHEGR emission, the anticipated EGRB is consis-

\footnotetext{
19 Note that $\phi_{B}(z, L)$ was determined assuming an unbroken spectrum, and thus in this case would over estimate the total flux from the TeV blazars. Nevertheless, here this is not accounted for, i.e., we renormalize the broken powerlaw spectrum to our previous power-law estimate of the TeV blazar luminosity density. As a consequence, our estimates of the EGRB are also over estimates.
}
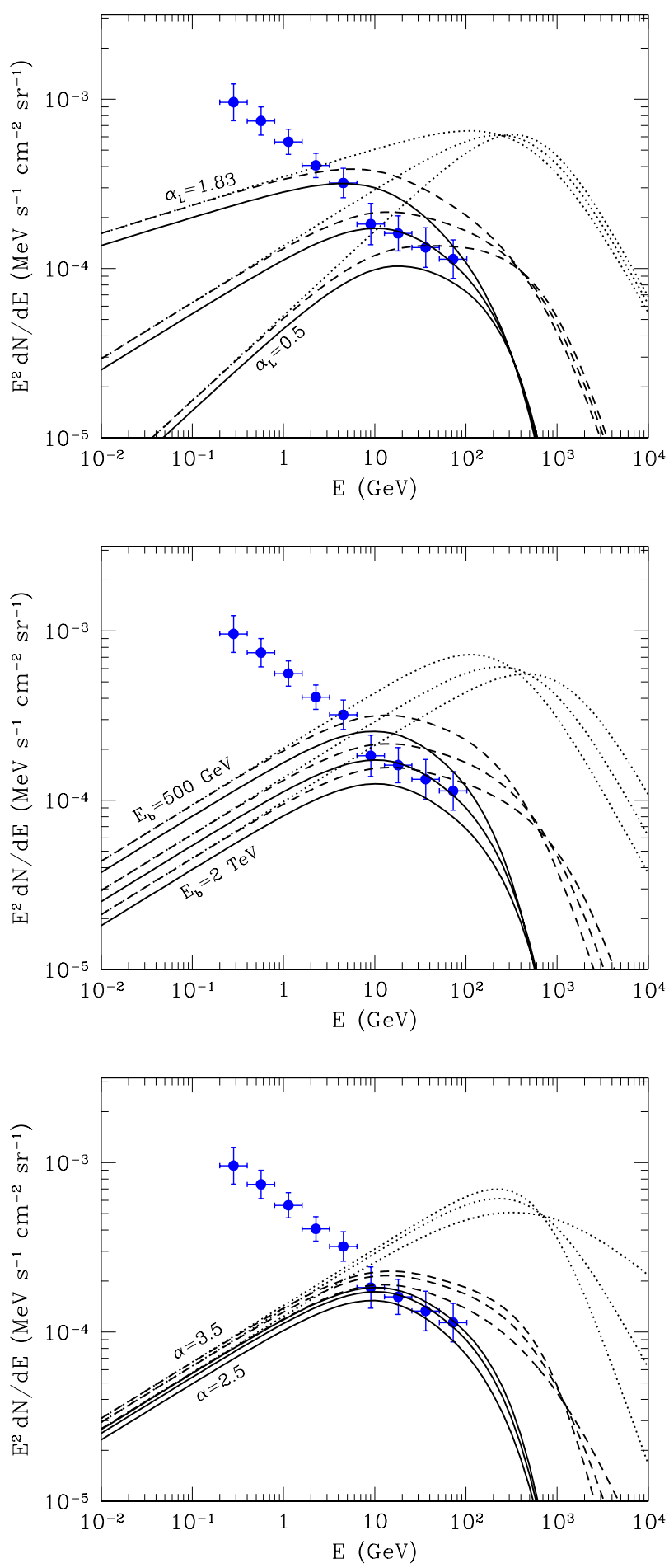

Figure 7. Contribution of TeV blazars to the EGRB. The solid lines show the contribution from TeV blazars beyond $z=0.25$, roughly the set for which Fermi can no-longer resolve them. The dashed lines show the contribution from all TeV blazars, including those which Fermi should have been able to detect individually and to remove. The dotted lines show the contribution from all TeV blazars when the annihilation upon the EBL is neglected. Different curves correspond to different parameters of the intrinsic spectrum assumed for the blazar populations. Top: Variations in the low-energy spectral index (top to bottom, $\left.\alpha_{L}=1.83,1.67,1.5\right)$. Middle: Variations in the break energy (top to bottom, $\left.E_{b}=500 \mathrm{GeV}, 1 \mathrm{TeV}, 2 \mathrm{TeV}\right)$. Bottom: Variations in the high-energy spectral index (top to bottom, $\alpha=3.5,3,3.5$ ). Unless otherwise specified, the remaining parameters are $\alpha_{L}=1.63, E_{b}=1 \mathrm{TeV}$, and $\alpha=3$. Finally, the Fermi measurement of the EGRB reported in Abdo et al. (2010a) is shown by the blue points. Note that in our scenario, the integral of the difference between the unabsorbed (dotted) and absorbed (dashed) energy fluxes, which dominates the total energy budget of these sources, has been dissipated into heat within the IGM. 
tent with the Fermi result. Thus, we find that despite our dramatically evolving $\phi_{B}$, we are are able to satisfy the limits imposed by the Fermi EGRB. Moreover, for reasonable spectralparameter values it is possible to accurately reproduce both the magnitude and shape of the high-energy EGRB (i.e., at energies $>10 \mathrm{GeV}) .{ }^{20}$ At first this may appear in conflict with other studies that have performed more sophisticated analyses of the gamma-ray emission from blazars (e.g., Narumoto \& Totani 2006; Kneiske \& Mannheim 2008; Inoue \& Totani 2009; Venters 2010) that have typically found that such a strongly evolving $\phi_{B}$ over-produces the Fermi EGRB. However, this is not the case for three reasons.

First, the AGN populations typically considered in computations of the EGRB include the FSRQs and LBLs, which dominate at low energies. The TeV blazars of interest here are only the high-energy tail of the blazar distribution, and it is these alone that we fix to the quasar luminosity function (indeed, it is for only these objects that the arguments surrounding Figure 5 have been made). Moreover, the TeV blazars themselves are generally dimmer, both bolometrically and within the Fermi LAT energy band $(200 \mathrm{MeV}-100 \mathrm{GeV})$, than the LBLs and FSRQs, which have typical isotropic-equivalent luminosities of $3 \times 10^{47} \mathrm{erg} \mathrm{s}^{-1}$ (Narumoto \& Totani 2006).

Second, and perhaps most importantly, the suppression of the ICCs means that the VHEGR emission is lost to heat in the IGM, not reprocessed to below $100 \mathrm{GeV}$. Since the TeV blazar flux is presumed to be dominated by emission above $100 \mathrm{GeV}$, the non-radiative dissipation of this emission substantially reduces the impact of the TeV blazars upon the EGRB. For all of the spectral parameters we considered the un-absorbed fluxes exceed the $30 \mathrm{GeV}$ EGRB by a significant margin, implying that the suppression of the ICCs is crucial to bringing the TeV blazar contributions in line with the Fermi EGRB. Thus, the lack of the ICCs generally appears necessary to reconcile the blazar and quasar luminosity functions.

Third, the VHEGR spectra from the most luminous TeV blazars necessarily peaks near or above $\mathrm{TeV}$ energies. For a number of the sources in Table 1, the intrinsic VHEGR spectrum (adjusted for annihilation on the EBL) is inverted, indicating that this break is above a TeV. The brightest $\mathrm{TeV}$ blazar in Table 1, Mkn 421, has an inverted spectrum below $100 \mathrm{GeV}$ (Abdo et al. 2010c), implying $E_{b} \sim 500 \mathrm{GeV}$. The second brightest, 1ES 1959+650, is consistent with being flat below $100 \mathrm{GeV}$, and appears to peak near $1 \mathrm{TeV}$ when in the highstate (Daniel et al. 2005). Similarly, 1ES 2344+514 peaks near $\sim 300 \mathrm{GeV}$ (Albert et al. 2007c) and Mkn 501 is inverted below $100 \mathrm{GeV}$, implying a peak above that value (Abdo et al. 2010c). Thus the sources likely to dominate the VHEGR background, and by extension the EGRB, all turn over near $100 \mathrm{GeV}-1 \mathrm{TeV}$. This is expected if the the VHEGR emission arises from inverse-Compton scattering the synchrotron bump (see, e.g., Ghisellini 2011, and references therein). The consequence of the spectral break is the suppression of the contribution from the TeV blazars to the EGRB below $E_{b}$. Were the $\mathrm{TeV}$ blazar contribution to the EGRB dominated by sources with $E_{b} \lesssim 2 \times 10^{2} \mathrm{GeV}$ it would exceed the Fermi EGRB at $E \lesssim 10 \mathrm{GeV}$.

A more complete analysis of the EGRB should include a variety of spectra, including a distribution of break energies, smoothly connecting the HBL, IBL and LBL populations.

\footnotetext{
${ }^{20}$ Note that since we are only presenting the EGRB from the TeV blazars, we necessarily neglect the contributions from the FSRQs and LBLs which dominate the EGRB at low energies.
}

However, this is beyond the scope of this paper, which is primarily concerned with the fate of the VHEGR emission, absent in the LBLs and FSRQs. Nevertheless a recent comprehensive model, which includes the contributions of the FSRQs and BL Lacs observed by Fermi, as well as starburst galaxies, has had considerable success fitting the Fermi EGRB with an even more extremely evolving $\phi_{B}$; fixing it to the luminosity function of radio galaxies (Cavadini et al. 2011; Stecker \& Venters 2011). Of particular relevance here is that Cavadini et al. (2011) and Stecker \& Venters (2011) explicitly ignored the potential contributions of ICCs. Unlike their analysis, however, we find that the $\mathrm{TeV}$ blazars are capable of reaching the highest Fermi bands despite the annihilation upon the EBL.

\section{CONCLUSIONS}

The cold, highly anisotropic beams of ultra-relativistic $e^{ \pm}$ pairs produced by the annihilation of VHEGRs upon the EBL are unstable to plasma beam instabilities. More importantly, for a wide range of parameters relevant for the observed $\mathrm{TeV}$ blazars these instabilities may be capable of isotropizing, and potentially extracting the kinetic energy of, the pairs at a rate orders of magnitude faster than inverse-Compton scattering.

This has far reaching consequences for efforts to constrain the IGMF using empirical limits upon the GeV emission from known TeV sources. Typically, $\sim 300 \mathrm{yr}$ after the onset of $\mathrm{TeV}$ emission the pair beam density has grown sufficiently for plasma beam instabilities to dominate its evolution, randomize the beam, and potentially suppress the inverse-Compton signal upon which the IGMF limits are based. Note that due to the beam disruption by the instabilities, this occurs even if the plasma instabilities do not ultimately cool the pairs. As a consequence, the present constraints upon the IGMF, obtained by the non-observation of an inverse-Compton GeV bump in the spectra of bright TeV blazars are inherently unreliable. Nevertheless, the sudden appearance of a TeV-bright blazar or intrinsically transient sources (e.g., GRBs) provide a means to temporarily avoid the consequences of plasma beam instabilities during the growth of the pair beam. Alternatively, observing particularly dim sources, $L \lesssim 10^{42} \mathrm{erg} \mathrm{s}^{-1}$, limits the beam density directly, again avoiding the complications imposed by plasma processes. Finally, the presence of these plasma instabilities in the pair beams of TeV blazars, which manifest themselves through their impact on the IGM (see Paper II, Paper III, and Puchwein et al. (2011)), implies the most stringent upper limit to date on the IGMF: $B \lesssim 10^{-12} \mathrm{G}$.

If the plasma instabilities can efficiently convert the pair beam kinetic energy into heat in the IGM, as we anticipate based upon existing numerical simulations and the arguments in Section 3.5, they would necessarily suppress the development of ICCs, and thus prevent the reprocessing of the VHEGR emission from bright sources to $\mathrm{GeV}$ energies. The lack of ICCs, independent of the mechanism that facilitates the local dissipation of the pair kinetic energy, would greatly weaken the constraints upon the evolution of the blazar population derived from the unresolved EGRB measured by Fermi. By introducing a spectral break near $1 \mathrm{TeV}$ and eliminating the reprocessed VHEGR emission, we find that the Fermi EGRB is consistent with a TeV blazar (and therefore presumably hard gamma-ray blazar) luminosity function fixed to that of quasars, normalized by comparing objects in the local Universe $(z \simeq 0.1)$, and motivated by the remarkable similarity between them in the local Universe. This conclusion is relatively insensitive to the particular parameters governing the VHEGR spectra of the TeV 
blazars, requiring only that the VHEGR emission is produced via inverse-Compton scattering. For a wide range of spectral parameters, we are able to match the magnitude and shape of the Fermi EGRB at high energies, with the low-energy component presumably arising from the FSRQs and LBLs. This $\phi_{B}$, and perhaps an even more rapidly evolving luminosity function, is also consistent with the observed redshift distribution of the 1LAC HSP and hard-ISP sample.

Matching the high-energy EGRB (above $10 \mathrm{GeV}$ ) requires more TeV blazars than are currently observed, though a comparable number to those inferred once the sky-coverage and $\mathrm{GeV}$ duty-cycle completeness corrections are included. Based upon these factors, and our rough estimate of the EGRB, we predict that upcoming surveys performed with nextgeneration Cerenkov telescope arrays (see, e.g., CTA Consortium 2010) should find roughly $2 \times 10^{2}$ sources above $4.2 \times$ $10^{-12} \mathrm{erg} \mathrm{s}^{-1} \mathrm{~cm}^{-2}$ (our estimate of the effective flux limit of current imaging Cerenkov telescopes), and a handful of additional sources comparable to the brightest $\mathrm{TeV}$ blazars observed. In addition, based upon the current number of known TeV blazars and our estimate of $\phi_{B}(z, L)$, the improved anticipated sensitivities of these instruments, 5-10 time larger than current arrays, should result in the detection of $1.5 \times 10^{3}-3 \times 10^{3}$ additional TeV blazars, with median luminosities $\sim 3 \times 10^{45} \mathrm{erg} \mathrm{s}^{-1}$. These should allow more precise estimates for their gammaray SEDs and a better characterization of $\phi_{B}(z, L)$, especially for low-luminosity objects.

Unlike inverse-Compton cooling, the plasma beam instabilities deposit the energy locally, heating the IGM. Moreover, the homogeneity of the EBL, and the weak dependence of the plasma cooling rates upon the IGM density, result in a uniform volumetric heating, in clear contrast to either photoionization heating or mechanical feedback from AGN. While we shall defer a detailed discussion of the consequences of this heating to Papers II, III, and Puchwein et al. (2011) here we note that this unusual heating prescription naturally explains a number of heretofore outstanding questions, including the inverted equation-of-state (temperature-density relation) for low density regions in the IGM (Paper II), the suppression of dwarf galaxies and their histories, the segregation of galaxy clusters and groups into cool core and non-cool core populations (Paper III), and the quantitative properties of the high-redshift Ly $\alpha$ forest (Puchwein et al. 2011). As a consequence, despite the fact that our estimates of the plasma cooling rates are limited to the linear regime (though with some numerical support), there are a variety of observational reasons to believe that plasma cooling, or an analogous mechanism, does in fact dominate the evolution of the ultra-relativistic pair beams.

We thank Tom Abel, Marco Ajello, Marcelo Alvarez, Arif Babul, Roger Blandford, James Bolton, Mike Boylan-Kolchin, Luigi Costamante, Andrei Gruzinov, Peter Goldreich, Martin Haehnelt, Andrey Kravtsov, Ue-li Pen, Ewald Puchwein, Volker Springel, Chris Thompson, Matteo Viel, Marc Voit, and Risa Wechsler for useful discussions. We are indebted to Peng Oh for his encouragement and useful suggestions. We thank Steve Furlanetto for kindly providing technical expertise. These computations were performed on the Sunnyvale cluster at CITA. A.E.B. and P.C. are supported by CITA. A.E.B. gratefully acknowledges the support of the Beatrice D. Tremaine Fellowship. C.P. gratefully acknowledges financial support of the Klaus Tschira Foundation and would furthermore like to thank KITP for their hospitality during the galaxy cluster work- shop. This research was supported in part by the National Science Foundation under Grant No. NSF PHY05-51164.

\section{APPENDIX}

\section{A. INSTABILITY GROWTH RATES}

Here we compute the growth rates for the various plasma instabilities discussed in the text within the context of relativistically moving pair plasmas. In all cases we make use of the kinetic theory description of the underlying plasmas. This necessarily assumes that the plasma, and in particular the beam plasma, is sufficiently dense that it is well described by a distribution function on the relevant scales. This is equivalent to requiring that many particles within a characteristic energy range be present on the plasma scale of the IGM, i.e., the conditions laid out in Section 3.1. In our analysis the Vlasov equation will play a central role, which owing to the relativistic nature of the calculation, we express in terms of the canonical pair, $\mathbf{x}$ and $\mathbf{p}$, making use of the Lorentz invariance of the electron/positron distribution functions $f^{\mp}(\mathbf{x}, \mathbf{p})$ and the phase-space volume element $d^{3} x d^{3} p$. In what follows we set $c=1$ unless otherwise specified.

\section{A.1. Relativistic Pair Two-Stream Instability}

The two-stream instability arises due to the excitation of negative-energy electrostatic waves in the beam and target plasmas. These waves carry away both the energy and momentum of the beam. Specifically, we compute the growth rate of the electrostatic wave moving in the direction of the beam in the absence of a background magnetic field ${ }^{21}$. In this case, the Vlasov equations for the electrons and positrons are:

$$
\begin{aligned}
& \frac{D f^{-}}{D t}-e \mathbf{E} \cdot \frac{\partial f^{-}}{\partial \mathbf{p}}=0 \\
& \frac{D f^{+}}{D t}+e \mathbf{E} \cdot \frac{\partial f^{+}}{\partial \mathbf{p}}=0
\end{aligned}
$$

where $D / D t \equiv \partial / \partial t+\mathbf{v} \cdot \boldsymbol{\nabla}$ is the Stokes derivative and $\mathbf{E}$ is the net electric field. Linearizing these and Fourier transforming in $t$ and $\mathbf{x}$ gives

$$
\begin{aligned}
& -i(\omega-\mathbf{k} \cdot \mathbf{v}) f_{1}^{-}-e \mathbf{E}_{1} \cdot \frac{\partial f_{0}^{-}}{\partial \mathbf{p}}=0 \\
& -i(\omega-\mathbf{k} \cdot \mathbf{v}) f_{1}^{+}+e \mathbf{E}_{1} \cdot \frac{\partial f_{0}^{+}}{\partial \mathbf{p}}=0
\end{aligned}
$$

where $f_{1}^{\mp}$ are the perturbations to the electron and positron distribution functions, $\mathbf{E}_{1}$ is the electrostatic wave field and we have assumed that the background distributions, $f_{0}^{\mp}$, are isotropic. As a result we have

$$
f_{1}^{\mp}=\frac{ \pm i e}{\omega-\mathbf{k} \cdot \mathbf{v}} \mathbf{E}_{1} \cdot \frac{\partial f_{0}^{ \pm}}{\partial \mathbf{p}} .
$$

At this point we may compute the dielectric tensor associated with the plasma response, however it will suffice to consider Gauss's law. Thus we now compute the perturbed charge

\footnotetext{
${ }^{21}$ Where the beam energy density is large in comparison to that associated with the background magnetic field this is well justified.
} 
density:

$$
\begin{aligned}
\rho_{1} & =\int\left(e f_{1}^{+}-e f_{1}^{-}\right) \mathrm{d}^{3} p \\
& =-i e^{2} \mathbf{E}_{1} \cdot \int \frac{1}{\omega-\mathbf{k} \cdot \mathbf{v}}\left(\frac{\partial f_{0}^{-}}{\partial \mathbf{p}}+\frac{\partial f_{0}^{+}}{\partial \mathbf{p}}\right) \mathrm{d}^{3} p \\
& =i e^{2} \mathbf{E}_{1} \cdot \int\left(f_{0}^{-}+f_{0}^{+}\right)\left(\frac{\partial}{\partial \mathbf{p}} \frac{1}{\omega-\mathbf{k} \cdot \mathbf{v}}\right) \mathrm{d}^{3} p \\
& =\frac{i e^{2}}{m_{e}} \mathbf{E}_{1} \cdot \int\left(f_{0}^{-}+f_{0}^{+}\right) \frac{(\mathbf{k}-\mathbf{k} \cdot \mathbf{v v})}{\gamma(\omega-\mathbf{k} \cdot \mathbf{v})^{2}} \mathrm{~d}^{3} p .
\end{aligned}
$$

It is now necessary to specify the $f_{0}^{\mp}$. We idealize the target ionic plasma as cold and the pair beam plasma as monoenergetic, yielding

$$
f_{0}^{-}=n_{t} \delta^{3}(\mathbf{p})+\frac{n_{b}}{2} \delta^{3}\left(\mathbf{p}-\mathbf{p}_{b}\right) \text { and } f_{0}^{+}=\frac{n_{b}}{2} \delta^{3}\left(\mathbf{p}-\mathbf{p}_{b}\right),
$$

where $n_{t}$ and $n_{b}$ are the lepton number densities in the target and beam, respectively. After performing the trivial integrals we then obtain

$$
\begin{aligned}
\rho_{1} & =\frac{i e^{2}}{m_{e}} \mathbf{E}_{1} \cdot\left(n_{t} \frac{\mathbf{k}}{\omega^{2}}+n_{b} \frac{\mathbf{k}-\mathbf{k} \cdot \mathbf{v}_{b} \mathbf{v}_{b}}{\gamma_{b}\left(\omega-\mathbf{k} \cdot \mathbf{v}_{b}\right)^{2}}\right) \\
& =\frac{i e^{2}}{m_{e}} \mathbf{E}_{1} \cdot \mathbf{k}\left(\frac{n_{t}}{\omega^{2}}+\frac{n_{b}}{\gamma_{b}^{3}\left(\omega-k v_{b}\right)^{2}}\right),
\end{aligned}
$$

were in the final equality we used the fact that $\mathbf{k} \| \mathbf{v}_{b}$. From Gauss's law we have $i \mathbf{k} \cdot \mathbf{E}_{1}=4 \pi \rho_{1}$, and therefore

$$
1-\frac{\omega_{P, t}^{2}}{\omega^{2}}-\frac{\omega_{P, b}^{2}}{\gamma_{b}^{3}\left(\omega-k v_{b}\right)^{2}}=0
$$

where $\omega_{P, t}^{2} \equiv 4 \pi e^{2} n_{t} / m_{e}$ and $\omega_{P, b}^{2} \equiv 4 \pi e^{2} n_{b} / m_{e}$ are the plasma frequencies associated with the target and beam plasmas.

This explicitly provides the dispersion relation, quadratic in $\omega$ (one electrostatic wave traveling in each direction for each plasma). When $n_{b}=0, \omega=\omega_{P, t}$. When $n_{b} \ll n_{t}$, as is the case of interest here, we may solve the dispersion relation perturbatively. We do this by setting $\omega=\omega_{P, t}(1+\eta)$, with $\eta \ll 1$, which gives:

$$
2 \eta-\frac{\omega_{P, b}^{2}}{\gamma_{b}^{3}\left(\omega_{P, t}-k v_{b}+\eta \omega_{P, t}\right)^{2}}=0,
$$

which is no longer independent of $k$. When $\left|\omega_{P, t}-k v_{b}\right| \gg$ $\left|\eta \omega_{P, t}\right|, \eta$ is real and thus there is no instability. On the other hand, where $k \simeq \omega_{P, t} / v_{b}$, we have

$$
2 \eta-\frac{\omega_{P, b}^{2}}{\gamma_{b}^{3} \omega_{P, t}^{2} \eta^{2}}=0
$$

and therefore $\eta^{3}=n_{b} / 2 n_{t} \gamma_{b}^{3}$. This has three solutions:

$$
\eta=\frac{1}{\gamma_{b}}\left(\frac{n_{b}}{2 n_{t}}\right)^{1 / 3}\left\{1, \quad-\frac{1}{2}-\frac{\sqrt{3}}{2} i, \quad-\frac{1}{2}+\frac{\sqrt{3}}{2} i\right\}
$$

the first of which is oscillatory, the second is decaying and the third is growing with timescale

$$
\Im(\omega)=\Im\left(\omega_{P, t} \eta\right)=\frac{\sqrt{3}}{2}\left(\frac{n_{b}}{2 n_{t}}\right)^{1 / 3} \frac{\omega_{P, t}}{\gamma_{b}} .
$$

This differs from that associated with non-relativistic, ionic beams only by the factor of $1 / \gamma_{b}$, arising due to time-dilation within the beam.

Note that since the energy within the electrostatic wave is proportional to $E_{1}^{2}$, the rate at which energy is removed from the beam is $\Gamma_{\mathrm{TS}} \equiv 2 \Im(\omega)$.

\section{A.2. Relativistic Pair Weibel Instability}

The relativistic version of the Weibel instability has been discussed in some detail in the literature for the case of anisotropic, though symmetric beams (Weibel 1959; Fried 1959; Yoon \& Davidson 1987; Medvedev \& Loeb 1999). Here we consider a the case of a dilute pair beam incident upon a much denser target plasma. This is essentially identical to the two-stream instability discussed previously, though coupling instead to an electromagnetic mode with $\mathbf{k} \perp \mathbf{v}_{b}$.

Again we begin with the linearized Vlasov equations for the electrons and positrons:

$$
\begin{aligned}
& \frac{D f_{1}^{-}}{D t}-e\left(\mathbf{E}_{1}+\mathbf{v} \times \mathbf{B}_{1}\right) \cdot \frac{\partial f_{0}^{-}}{\partial \mathbf{p}}=0 \\
& \frac{D f_{1}^{+}}{D t}+e\left(\mathbf{E}_{1}+\mathbf{v} \times \mathbf{B}_{1}\right) \cdot \frac{\partial f_{0}^{+}}{\partial \mathbf{p}}=0,
\end{aligned}
$$

where we have $\mathbf{B}_{1}=\mathbf{k} \times \mathbf{E}_{1} / \omega$ from Faraday's law. Fourier transforming in $t$ and $\mathbf{x}$ and solving for $f_{1}^{\mp}$ gives

$$
\begin{aligned}
f_{1}^{\mp} & = \pm \frac{i e\left[\mathbf{E}_{1}+\mathbf{v} \times\left(\mathbf{k} \times \mathbf{E}_{1}\right) / \omega\right]}{\omega-\mathbf{k} \cdot \mathbf{v}} \cdot \frac{\partial f_{0}^{\mp}}{\partial \mathbf{p}} \\
& = \pm \frac{i e}{\omega}\left(\mathbf{E}_{1}+\frac{\mathbf{v} \cdot \mathbf{E}_{1}}{\omega-\mathbf{k} \cdot \mathbf{v}} \mathbf{k}\right) \cdot \frac{\partial f_{0}^{\mp}}{\partial \mathbf{p}} .
\end{aligned}
$$

The associated perturbation to the current is

$$
\begin{aligned}
\mathbf{j}_{1} & =\int\left(e f_{1}^{+}-e f_{1}^{-}\right) \mathbf{v} d^{3} p \\
& =-\frac{i e^{2}}{\omega} \int \frac{\partial\left(f_{0}^{+}+f_{0}^{-}\right)}{\partial \mathbf{p}} \cdot\left(\mathbf{E}_{1}+\frac{\mathbf{v} \cdot \mathbf{E}_{1}}{\omega-\mathbf{k} \cdot \mathbf{v}} \mathbf{k}\right) \mathbf{v} d^{3} p \\
& =\frac{i e^{2}}{\omega} \int \frac{f_{0}}{m_{e} \gamma}\left[\hat{\mathbf{1}}+\frac{\mathbf{k v}+\mathbf{v k}}{\omega-\mathbf{k} \cdot \mathbf{v}}-\frac{\omega^{2}-k^{2}}{(\omega-\mathbf{k} \cdot \mathbf{v})^{2}} \mathbf{v} \mathbf{v}\right] \cdot \mathbf{E}_{1} d^{3} p
\end{aligned}
$$

in which we've defined $f_{0} \equiv f_{0}^{+}+f_{0}^{-}$.

Choosing the $f_{0}^{\mp}$ given by Equation (A5) and assuming $\mathbf{k} \|$ $\mathbf{v}_{b} \| \mathbf{E}_{1}$ we recover the standard two-stream instability. For our purposes here, however, the computations may be substantially simplified by boosting into a frame in which the target plasma is not at rest. In this frame we have $f_{0}$ given by,

$$
f_{0}=n_{t}^{\prime} \delta^{3}\left(\mathbf{p}^{\prime}-\mathbf{p}_{t}^{\prime}\right)+n_{b}^{\prime} \delta^{3}\left(\mathbf{p}^{\prime}-\mathbf{p}_{b}^{\prime}\right)
$$

with $\mathbf{p}_{t}^{\prime}, \mathbf{p}_{b}^{\prime} \| \mathbf{v}_{b}^{\prime}$, where we will take care at the end to properly relate all of these quantities to their target-frame analogs. In particular, we choose this frame such that $\tilde{n}_{t} v_{t}^{\prime}=\tilde{n}_{b} v_{b}^{\prime}$, where $\tilde{n}_{t}$ and $\tilde{n}_{b}$ are the proper densities of the target and beam plasmas, respectively. If $v_{b}$ is the beam plasma velocity in the target (or lab) frame, this gives

$$
v_{t}^{\prime}=\xi v_{b}^{\prime}=\xi \frac{v_{b}-v_{t}^{\prime}}{1-v_{b} v_{t}^{\prime}}
$$

where $\xi \equiv \tilde{n}_{b} / \tilde{n}_{t}$ and is in our case much less than unity. Note that this is only the center of momentum frame if $\xi=1$ (for which $v_{t}^{\prime}=v_{b}^{\prime}$, the case most commonly discussed). If we 
choose $\mathbf{k}^{\prime} \perp \mathbf{v}_{b}^{\prime}$ this choice of frame causes the terms linear in $\mathbf{v}_{b}^{\prime}$ in Equation (A14) to vanish identically, yielding

$\mathbf{j}_{1}^{\prime}=\frac{i e^{2}}{m_{e} \omega^{\prime}}\left[\left(\frac{n_{t}^{\prime}}{\gamma_{t}^{\prime}}+\frac{n_{b}^{\prime}}{\gamma_{b}^{\prime}}\right) \hat{\mathbf{1}}-\frac{\omega^{\prime 2}-k^{\prime 2}}{\omega^{\prime 2}}\left(\frac{n_{t}^{\prime}}{\gamma_{t}^{\prime}} \mathbf{v}_{t}^{\prime} \mathbf{v}_{t}^{\prime}+\frac{n_{b}^{\prime}}{\gamma_{b}^{\prime}} \mathbf{v}_{b}^{\prime} \mathbf{v}_{b}^{\prime}\right)\right] \cdot \mathbf{E}_{1}^{\prime}$.

Using the inhomogeneous wave equation, obtained from Faraday's and Ampere's law,

$$
\left(k^{\prime 2}-\omega^{\prime 2}\right) \mathbf{E}_{1}^{\prime}=4 \pi i \omega^{\prime} \mathbf{j}_{1}^{\prime},
$$

and choosing $\mathbf{E}_{1}^{\prime} \| \mathbf{v}_{b}^{\prime}$ produces the dispersion relation

$$
\omega^{\prime 2}-k^{\prime 2}-\frac{\omega_{P, t}^{\prime 2}}{\gamma_{t}^{\prime 3}}-\frac{\omega_{P, b}^{\prime 2}}{\gamma_{b}^{\prime 3}}-\frac{k^{\prime 2}}{\omega^{\prime 2}}\left(\frac{\omega_{P, t}^{\prime 2} v_{t}^{\prime 2}}{\gamma_{t}^{\prime}}+\frac{\omega_{P, b}^{\prime 2} v_{b}^{\prime 2}}{\gamma_{b}^{\prime}}\right)=0 \text {. }
$$

At this point we make use of the fact that in our case $\xi \ll 1$, which allows a perturbative solution of Equation (A16): $v_{t}^{\prime}=$ $\xi v_{b}+\mathcal{O}\left(\xi^{2}\right)$ and therefore $v_{b}^{\prime}=v_{b}(1-\xi)+\mathcal{O}\left(\xi^{2}\right)$. Furthermore, $\omega^{\prime}=\omega+\mathcal{O}\left(\xi^{2}\right)$ and $k^{\prime}=k$ for the geometry under consideration. As consequence, $\omega_{P, t}^{\prime} v_{t}^{\prime 2} / \gamma_{t}^{\prime} \simeq \omega_{P, t} \xi^{2} v_{b}^{2}=\xi \omega_{P, b} v_{b}^{2} / \gamma_{b} \ll$ $\omega_{P, b} v_{b}^{2} / \gamma_{b}$ and $\omega_{P, t}^{\prime 2} / \gamma_{t}^{\prime 3} \simeq \omega_{P, t}^{2}=\xi^{-1} \omega_{P, b}^{2} \gg \omega_{P, b}^{\prime 2} / \gamma_{b}^{\prime 3}$, which implies that

$$
\omega^{2}-k^{2}-\omega_{P, t}^{2}-\frac{k^{2}}{\omega^{2}} \frac{\omega_{P, b}^{2} v_{b}^{2}}{\gamma_{b}}(1-2 \xi)=0 .
$$

This has a purely imaginary solution:

$$
\omega=i \sqrt{\frac{k^{2}+\omega_{P, t}^{2}}{2}} \sqrt{\left[1+\frac{4 k^{2} \omega_{P, b}^{2} v_{b}^{2}(1-2 \xi)}{\left(k^{2}+\omega_{P, t}^{2}\right)^{2} \gamma_{b}}\right]^{1 / 2}-1} .
$$

For $k \ll \omega_{P, t}$ this rises linearly with $k$, saturating for $k>\omega_{P, t} \gg$ $\omega_{P, b} v_{b}^{2} / \sqrt{\gamma_{b}}$, giving the growth rate

$$
\Im(\omega) \simeq \frac{\omega_{P, b} v_{b}}{\sqrt{\gamma_{b}}}(1-\xi)+\mathcal{O}\left(\xi^{2}\right) .
$$

Given that for our application $\xi \ll 1$, we will drop terms first order in $\xi$ as well. Note that the plasma frequency that enters into the growth rate is that of the beam, which is much lower than that associated with the target plasma. Nevertheless, the scale of the rapidly growing perturbations is limited to that associated with the target plasma, which are general much smaller than that associated with the perturbations in the beam alone. Again, the rate at which energy is sapped from the beam is then $\Gamma_{\mathrm{W}}=2 \Im(\omega)$.

\section{B. AN EXPLICIT EXPRESSION FOR THE QUAS AR LUMINOSITY FUNCTION}

In the interests of completeness, here we reproduce the $\phi_{Q}$ from Hopkins et al. (2007), corresponding to the "Full" case in that paper, that we employ. See Hopkins et al. (2007) for how this $\phi_{Q}$ was obtained, and caveats regarding its application.

The form of $\phi_{Q}$ is assumed to be a broken power law:

$$
\phi_{Q}(z, L)=\frac{\phi_{*}}{\left[L / L_{*}(z)\right]^{\gamma_{1}(z)}+\left[L / L_{*}(z)\right]^{\gamma_{2}(z)}},
$$

where the location of the break $\left(L_{*}(z)\right)$ and the power laws $\left(\gamma_{1}(z)\right.$ and $\left.\gamma_{2}(z)\right)$ are functions of redshift. These are given by,

$$
\begin{aligned}
\log _{10} L_{*}(z) & =\left(\log _{10} L_{*}\right)_{0}+k_{L, 1} \xi+k_{L, 2} \xi^{2}+k_{L, 3} \xi^{3} \\
\gamma_{1}(z) & =\gamma_{1,0} 10^{k_{\gamma_{1}}} \xi \\
\gamma_{2}(z) & =2 \gamma_{2,0}\left(10^{k_{\gamma_{2}, 1}} \xi+10^{k_{\gamma_{2}, 2} \xi}\right)^{-1}
\end{aligned}
$$



Figure 8. Cumulative (top) and differential (bottom) number of clean Fermi HSPs as a function of measured spectral index. Different line colors correspond to different subpopulations of the Fermi HSP sample: all HSPs with measured redshifts (green), HSPs with measured redshifts $>0.25$ (red), HSPs with measured redshifts $<0.25$ (blue), and HSPs without redshift measurements (black). In the bottom panel, error bars denote the Poisson uncertainty only.

where

$$
\xi \equiv \log _{10}\left(\frac{1+z}{3}\right) .
$$

The values of the relevant parameters are given in Table 2, and where any estimate of the uncertainty is made we assume these are independently, normally distributed.

\section{INFERRING THE REDSHIFT DISTRIBUTION OF THE FERMI HSPS WITHOUT MEASURED REDSHIFTS}

Of the 118 HSPs detected by Fermi and reported in the First LAT AGN Catalog (1LAC, Abdo et al. 2010c, , there called HSPs), 113 are members of the clean sample (meaning that there are no ambiguities surrounding their detection), and of these only 65 have measured redshifts. This is a somewhat larger fraction that for 1LAC BL Lacs generally, though this leaves nearly half of the HSP population with their redshifts undetermined. Based upon comparisons between the spectral index and flux distributions between the HSPs without redshifts and those at various redshift ranges, here we argue that these are likely to be located nearby.

Already, based upon comparisons between the spectral index distributions (SIDs) of the 1LAC BL Lacs at large, it is clear that the objects with and without measured redshifts are not drawn from the same underlying population (Abdo et al. 2010c). Similarities between the SIDs of the unknown- $z$ objects and the $z>0.5$ subset of those with measured redshifts, Abdo et al. (2010c) has suggested that the BL Lacs without redshifts may be biased towards higher redshifts. However, we note that there are only three HSPs with $z>0.5$, and thus this conclusion is relevant for ISPs and LSPs only.

The SIDs of the unknown- $z$ HSPs is shown in Figure 8, compared with the SIDs of HSPs with redshifts (cf. Figure 22 of Abdo et al. 2010c). The Kolmogorov-Smirnov (KS) proba- 
Table 2

Parameters of the Quasar Luminosity Function from Hopkins et al. (2007)

\begin{tabular}{|c|c|c|c|c|c|c|}
\hline \multicolumn{2}{|c|}{ Normalization } & \multicolumn{2}{|c|}{$\log _{10} L_{*}$} & \multicolumn{2}{|r|}{$\gamma_{1}$} & $\gamma_{2}$ \\
\hline $\log _{10} \phi_{*}{ }^{a}$ & $-4.825 \pm 0.060$ & $\begin{array}{c}\left(\log _{10} L_{*}\right)_{0} \mathrm{~b} \\
k_{L, 1} \\
k_{L, 2} \\
k_{L, 3}\end{array}$ & $\begin{array}{l}13.036 \pm 0.043 \\
0.632 \pm 0.077 \\
-11.76 \pm 0.38 \\
-14.25 \pm 0.80\end{array}$ & $\begin{array}{l}\gamma_{1,0} \\
k_{\gamma_{1}}\end{array}$ & $\begin{array}{c}0.417 \pm 0.055 \\
-0.623 \pm 0.132\end{array}$ & $\begin{array}{cc}\gamma_{2,0} & 2.174 \pm 0.055 \\
k_{\gamma_{2}, 1} & 1.460 \pm 0.096 \\
k_{\gamma_{2}, 2} & -0.793 \pm 0.057\end{array}$ \\
\hline
\end{tabular}

In units of comoving $\mathrm{Mpc}^{-3}$

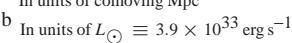



Figure 9. Cumulative (top) and differential (bottom) number of clean Fermi HSPs as a function of integrated flux between $1 \mathrm{GeV}$ and $100 \mathrm{GeV}$. Different line colors correspond to different subpopulations of the Fermi HSP sample: all HSPs with measured redshifts (green), HSPs with measured redshifts $>0.25$ (red), HSPs with measured redshifts $<0.25$ (blue), and HSPs without redshift measurements (black). In the bottom panel, error bars denote the Poisson uncertainty only.

Table 3

Comparison Between HSPs With and Without Measured Redshifts

\begin{tabular}{lcccc}
\hline \hline \multicolumn{1}{c}{ Redshift } & \multicolumn{2}{c}{ Spectral Index } & \multicolumn{2}{c}{ Flux between $1 \mathrm{GeV}-100 \mathrm{GeV}$} \\
\multicolumn{1}{c}{ Population } & $P_{\mathrm{KS}}$ & $\langle\alpha\rangle$ & $P_{\mathrm{KS}}$ & $\left\langle\log _{10}\left(F_{35} / \mathrm{cm}^{-2} \mathrm{~s}^{-1}\right)\right\rangle$ \\
\hline All HSPs with $z$ 's & 0.04 & $2.84 \pm 0.03$ & 0.07 & $-8.89 \pm 0.01$ \\
HSPs with $z \geq 0.25$ & 0.01 & $2.83 \pm 0.05$ & 0.007 & $-9.06 \pm 0.03$ \\
HSPs with $z<0.25$ & 0.45 & $2.84 \pm 0.03$ & 0.42 & $-8.78 \pm 0.02$ \\
HSPs without $z$ 's & - & $2.91 \pm 0.03$ & - & $-8.86 \pm 0.02$ \\
& & & & \\
\hline
\end{tabular}

bility that these are drawn from the same parent population is 0.04 , indicating that this is unlikely at the $2-\sigma$ level. In addition, we show the SIDs of HSPs with $z<0.25$ (blue line) and $z \geq 0.25$ (red line), with corresponding KS probabilities of 0.45 and 0.01 , respectively (these are collected in Table 3 ). Thus, in contrast to the 1LAC BL Lac sample at large, the HSPs without redshifts appear to have SIDs that are strongly inconsistent with the population observed to have high redshifts, and indistinguishable from those at low redshifts. Nevertheless, the HSPs with unknown-zs still tend to be softer than those with

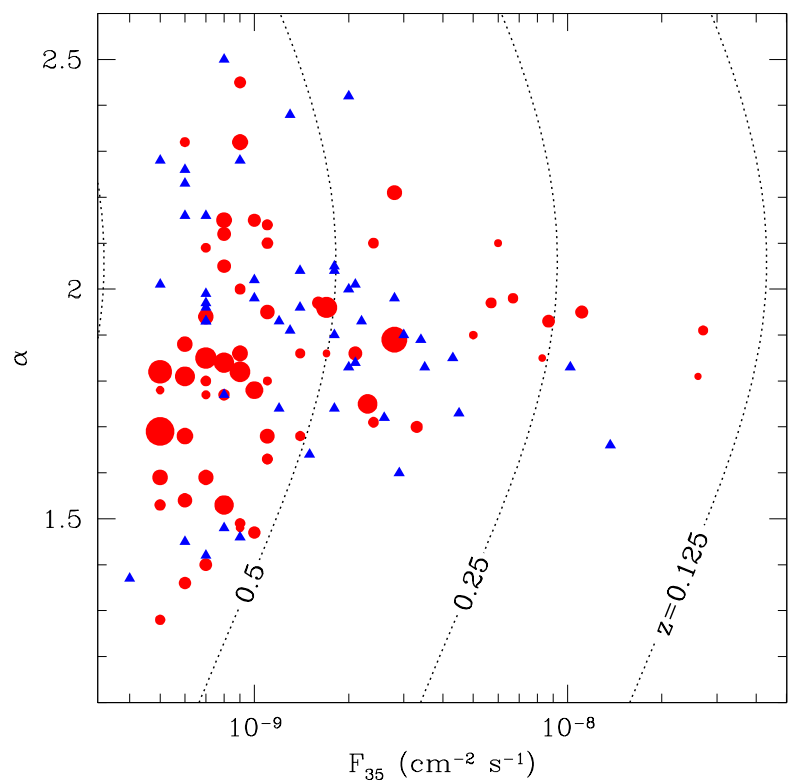

Figure 10. Redshift and spectral index-flux distribution of the clean Fermi HSP sample. HSPs with measured redshifts are shown by the red circles, with the circle size linearly related to $z$ (ranging between $z=0$ and 0.7 , i.e., large $z$ is denoted by large points and small $z$ by small points). HSPs without redshifts are shown by the blue triangles. For reference, the dotted black lines show lines of constant redshift for a source with luminosity $2 \times 10^{46} \mathrm{erg} \mathrm{s}^{-1}$ between $100 \mathrm{MeV}$ and $100 \mathrm{GeV}$ (the definition of $L_{\gamma}$ in Abdo et al. 2010c, note the difference with the definition of $F_{35}$ ).

measured redshifts in either range.

A similar analysis may be performed upon the reported flux measure, $F_{35}$, corresponding to the flux between $1 \mathrm{GeV}$ and $100 \mathrm{GeV}$. Figure 9 shows the flux distributions (FDs) of the unknown- $z$, all measured $z, z \geq 0.25$, and $z<0.25$ HSP populations, and is analogous to Figure 8. As before, the HSPs without redshifts have FDs that are marginally inconsistent with being drawn from the same parent population as the complete set of HSPs with measured redshifts, having a KS probability of 0.07. The inconsistency with the high- $z$ HSP FD is even more striking than for the SIDs, with a KS probability less than 0.007 , i.e., that the high- $z$ FD of the unknown- $z$ and $z \geq 0.25$ HSPs are from the same distribution is excluded. However, again, we find that the FDs of the low $-z$ and unknown- $z$ populations are indistinguishable, having a KS probability of 0.42 .

That the SIDs and FDs both favor a relationship between the unknown- $z$ and low- $z$ HSP populations provides some confidence that this may, in fact, be the case. This is supported by the redshift distribution in the $\alpha-F_{35}$ plane, depicted in Figure 10. HSPs with high redshifts are clustered at low fluxes and hard $\alpha$. This is not unexpected given an upper limit upon the lu- 
minosity of HSPs. The dotted lines in Figure 10 show constant redshift curves in the $F_{35}-\alpha$ plane for a $100 \mathrm{MeV}-100 \mathrm{GeV}$ luminosity of $2 \times 10^{46} \mathrm{erg} \mathrm{s}^{-1}$ (the definition of $L_{\gamma}$ in Abdo et al. $2010 \mathrm{c}$, note the distinction with the definition of $F_{35}$ ). If all HSPs have intrinsic luminosities less than $2 \times 10^{46} \mathrm{erg} \mathrm{s}^{-1}$, no sources at a given $z$ should be found to the right of the associated line. Since the volume of the visible Universe is dominated by $z \sim 1$, the majority of high- $z$ objects should then be found up against the instrumental flux limit, i.e., at low fluxes and nearly flat spectra. Sources with harder spectra are likely to have higher bolometric gamma-ray luminosities since they are likely to be more below the inverse-Compton peak, biasing high- $z$ sources towards harder spectra.

However, the HSPs without redshifts are noticeably absent within this high- $z$ dominated region. This is responsible for the fact that they more closely share their SID and FD with the low- $z$ HSPs. The presence of HSPs without redshifts at a variety of $\alpha \mathrm{s}$ and $F_{35} \mathrm{~s}$ suggests that this is not a result of a selection effect upon either. It is nonetheless possible that there is some instrumental effect which prevents the measurement of high redshifts in intrinsically bright, soft objects, in which case a population of high-luminosity HSPs with high (and therefore unmeasured) $z$ s would necessarily be located in regions with predominantly low measured $z \mathrm{~s}$. However, this is belied by the broad distribution of luminosities and spectral indexes among the HSPs with measured redshifts. Thus, we adopt the simpler explanation: the HSPs with unknown redshifts have and SID and FD similar to low- $z$ objects because they are intrinsically dim, nearby objects.

\section{REFERENCES}

Abdo, A. A., et al. 2009, ApJ, 700, 597

—. 2010a, Phys. Rev. Lett., 104, 101101

-. 2010b, ApJ, 720, 435

-. $2010 \mathrm{c}, \mathrm{ApJ}, 715,429$

-. 2010d, ApJ, 716, 30

Acciari, V., et al. 2009a, ApJ, 690, L126

Acciari, V. A., et al. 2008, ApJ, 679, 397

-. 2009b, ApJ, 707, 612

—. 2009c, ApJ, 693, L104

—. 2010a, ApJ, 709, L163

-. 2010b, ApJ, 708, L100

-. 2010c, ApJ, 715, L49

Aharonian, F., et al. 2002, A\&A, 384, L23

-. 2003, A\&A, 406, L9

-. 2005, A\&A, 436, L17

-. 2006, A\&A, 455, 461

-. 2007a, A\&A, 470, 475

-. 2007b, A\&A, 473, L25

-. 2007c, A\&A, 475, L9

-. 2008a, A\&A, 481, L103

-. 2008b, A\&A, 477, 481

-. 2010, A\&A, 521, 69

Albert, J., et al. 2006, ApJ, 648, L105

-. 2007a, ApJ, 666, L17

-. 2007b, ApJ, 667, L21

-. 2007c, ApJ, 662, 892

Aleksić, J., et al. 2010, ApJ, 723, L207

Aliu, E., et al. 2009, ApJ, 692, L29

Ando, S., \& Kusenko, A. 2010, ApJ, 722, L39

Barrow, J. D., Ferreira, P. G., \& Silk, J. 1997, Physical Review Letters, 78, 3610

Boyd, T. J. M., \& Sanderson, J. J. 2003, The Physics of Plasmas (Cambridge: Cambridge University Press)

Bret, A. 2009, ApJ, 699, 990

Bret, A., Firpo, M., \& Deutsch, C. 2004, Phys. Rev. E, 70, 046401

—. 2005a, Physical Review Letters, 94, 115002

—. 2005b, Phys. Rev. E, 72, 016403

Bret, A., Gremillet, L., \& Bénisti, D. 2010a, Phys. Rev. E, 81, 036402
Bret, A., Gremillet, L., \& Dieckmann, M. E. 2010b, Physics of Plasmas, 17, 120501

Cavadini, M., Salvaterra, R., \& Haardt, F. 2011, arXiv:1105.4613

Chandra, P., et al. 2010, Journal of Physics G Nuclear Physics, 37, 125201

Chang, P., Broderick, A. E., \& Pfrommer, C. 2012, ApJ in print, arXiv: 1106.5504

Chang, P., Spitkovsky, A., \& Arons, J. 2008, ApJ, 674, 378

Costamante, L., Aharonian, F., \& Khangulyan, D. 2007, in American Institute of Physics Conference Series, Vol. 921, The First GLAST Symposium, ed. S. Ritz, P. Michelson, \& C. A. Meegan, 157-159

CTA Consortium, T. 2010, arXiv:1008.3703

Dai, Z. G., \& Lu, T. 2002, ApJ, 580, 1013

Daniel, M. K., et al. 2005, ApJ, 621, 181

Davidson, R. C., Hammer, D. A., Haber, I., \& Wagner, C. E. 1972, Physics of Fluids, 15, 317

de Angelis, A., Persic, M., \& Roncadelli, M. 2008, Modern Physics Letters A, 23,315

Dermer, C. D., Cavadini, M., Razzaque, S., Finke, J. D., Chiang, J., \& Lott, B. 2011, ApJ, 733, L21

Dolag, K., Kachelriess, M., Ostapchenko, S., \& Tomàs, R. 2011, ApJ, 727, L4

Franceschini, A., Rodighiero, G., \& Vaccari, M. 2008, A\&A, 487, 837

Frederiksen, J. T., Hededal, C. B., Haugbølle, T., \& Nordlund, ̊. 2004, ApJ, 608, L13

Fried, B. D. 1959, Physics of Fluids, 2, 337

Ghisellini, G. 1999, Astrophysical Letters Communications, 39, 17

—. 2011, arXiv: 1104.0006

Ghisellini, G., Maraschi, L., \& Tavecchio, F. 2009, MNRAS, 396, L105

Ghisellini, G., \& Tavecchio, F. 2008, MNRAS, 387, 1669

Gould, R. J., \& Schréder, G. P. 1967, Physical Review, 155, 1408

Guetta, D., \& Granot, J. 2003, ApJ, 585, 885

HESS Collaboration et al. 2010, A\&A, 520, 83

Hopkins, P. F., Richards, G. T., \& Hernquist, L. 2007, ApJ, 654, 731

Huang, D., Konopelko, A., \& for the VERITAS collaboration. 2009, arXiv: 0912.3772

Inoue, Y., \& Totani, T. 2009, ApJ, 702, 523

Kandus, A., Kunze, K. E., \& Tsagas, C. G. 2011, Phys. Rep., 505, 1

Kneiske, T. M., Bretz, T., Mannheim, K., \& Hartmann, D. H. 2004, A\&A, 413, 807

Kneiske, T. M., \& Mannheim, K. 2008, A\&A, 479, 41

Komatsu, E., et al. 2011, ApJS, 192, 18

Kotera, K., \& Lemoine, M. 2008, Phys. Rev. D, 77, 123003

Lemoine, M., \& Pelletier, G. 2010, MNRAS, 402, 321

Lesch, H., \& Schlickeiser, R. 1987, A\&A, 179, 93

MAGIC Collaboration et al. 2008a, Science, 320, 1752

-. 2008b, Science, 320, 1752

Mariotti, M., \& MAGIC Collaboration. 2010, The Astronomer's Telegram, 2916,1

Massaro, E., Giommi, P., Leto, C., Marchegiani, P., Maselli, A., Perri, M., Piranomonte, S., \& Sclavi, S. 2009, A\&A, 495, 691

Medvedev, M. V., \& Loeb, A. 1999, ApJ, 526, 697

Melrose, D. B. 1980, Plasma astrophysics: Nonthermal processes in diffuse magnetized plasmas. Volume 2 - Astrophysical applications

Mose Mariotti, M. 2010, The Astronomer's Telegram, 2684, 1

Nakar, E., Bret, A., \& Milosavljevic, M. 2011, ArXiv 1104.5249

Narumoto, T., \& Totani, T. 2006, ApJ, 643, 81

Neronov, A., \& Semikoz, D. V. 2009, Phys. Rev. D, 80, 123012

Neronov, A., Semikoz, D. V., Tinyakov, P. G., \& Tkachev, I. I. 2011, A\&A, 526,90

Neronov, A., \& Vovk, I. 2010, Science, 328, 73

Oh, S. P. 2001, ApJ, 553, 25

Padovani, P., \& Giommi, P. 1995, ApJ, 444, 567

Pfrommer, C., Chang, P., \& Broderick, A. E. 2012, ApJ in print, arXiv: 1106.5505

Plaga, R. 1995, Nature, 374, 430

Puchwein, E., Pfrommer, C., Springel, V., Broderick, A. E., \& Chang, P. 2011, arXiv: 1107.3837

Raue, M., et al. 2010, in Astronomical Society of the Pacific Conference Series, Vol. 427, Astronomical Society of the Pacific Conference Series, ed. L. Maraschi, G. Ghisellini, R. Della Ceca, \& F. Tavecchio, 302

Salamon, M. H., \& Stecker, F. W. 1998, ApJ, 493, 547

Silva, L. O., Fonseca, R. A., Tonge, J. W., Dawson, J. M., Mori, W. B., \& Medvedev, M. V. 2003, ApJ, 596, L121

Spitkovsky, A. 2008, ApJ, 682, L5

Sreekumar, P., et al. 1998, ApJ, 494, 523

Stecker, F., \& Venters, T. M. 2011, ApJ, 736, 40

Stecker, F. W., de Jager, O. C., \& Salamon, M. H. 1992, ApJ, 390, L49 
Strong, A. W., Moskalenko, I. V., \& Reimer, O. 2004, ApJ, 613, 956

Sturrock, P. A. 1994, Plasma Physics, An Introduction to the Theory of Astrophysical, Geophysical and Laboratory Plasmas (Cambridge: Cambridge University Press)

Takahashi, K., Mori, M., Ichiki, K., \& Inoue, S. 2012, ApJ, 744, L7

Takahashi, K., Murase, K., Ichiki, K., Inoue, S., \& Nagataki, S. 2008, ApJ, 687, L5

Tavecchio, F., \& Ghisellini, G. 2008, MNRAS, 386, 945

Tavecchio, F., Ghisellini, G., Bonnoli, G., \& Foschini, L. 2011, MNRAS, 414, 3566

Tavecchio, F., Ghisellini, G., Foschini, L., Bonnoli, G., Ghirlanda, G., \& Coppi, P. 2010, MNRAS, 406, L70
Taylor, A. M., Vovk, I., \& Neronov, A. 2011, A\&A, 529, A144

Tramacere, A., Giommi, P., Perri, M., Verrecchia, F., \& Tosti, G. 2009, A\&A, 501,879

Urry, C. M., \& Padovani, P. 1995, PASP, 107, 803

Vallée, J. P. 2011, New Astron. Rev., 55, 91

Venters, T. M. 2010, ApJ, 710, 1530

Waxman, E., \& Miralda-Escude, J. 1996, ApJ, 472, L89+

Weibel, E. S. 1959, Physical Review Letters, 2, 83

Widrow, L. M. 2002, Reviews of Modern Physics, 74, 775

Yoon, P. H., \& Davidson, R. C. 1987, Phys. Rev. A, 35, 2718 